ARTICLE

https://doi.org/10.1038/s41467-020-14585-6

\title{
Precise timing of transcription by c-di-GMP coordinates cell cycle and morphogenesis in Caulobacter
}

Andreas Kaczmarczyk (10) 1,4, Antje M. Hempel (1) 1,4, Christoph von Arx (1) 1,3,4, Raphael Böhm (1) 2,

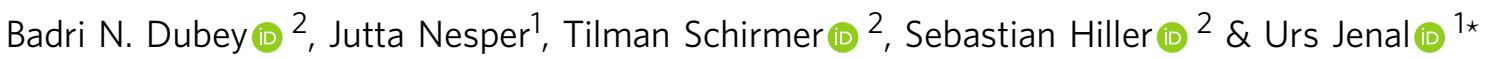

Bacteria adapt their growth rate to their metabolic status and environmental conditions by modulating the length of their G1 period. Here we demonstrate that a gradual increase in the concentration of the second messenger c-di-GMP determines precise gene expression during G1/S transition in Caulobacter crescentus. We show that c-di-GMP stimulates the kinase ShkA by binding to its central pseudo-receiver domain, activates the TacA transcription factor, and initiates a G1/S-specific transcription program leading to cell morphogenesis and S-phase entry. Activation of the ShkA-dependent genetic program causes c-di-GMP to reach peak levels, which triggers S-phase entry and promotes proteolysis of ShkA and TacA. Thus, a gradual increase of c-di-GMP results in precise control of ShkA-TacA activity, enabling G1/Sspecific gene expression that coordinates cell cycle and morphogenesis.

\footnotetext{
${ }^{1}$ Infection Biology, Biozentrum, University of Basel, Klingelbergstrasse 50, CH-4056 Basel, Switzerland. ${ }^{2}$ Structural Biology, Biozentrum, University of Basel Klingelbergstrasse 50, CH-4056 Basel, Switzerland. ${ }^{3}$ Present address: Interregional Blood Transfusion SRC Ltd., Murtenstrasse 133,3008 Bern, Switzerland.

${ }^{4}$ These authors contributed equally: Andreas Kaczmarczyk, Antje M. Hempel, Christoph von Arx. *email: urs.jenal@unibas.ch
} 
T he bacterial cell cycle is divided into three periods: after cell division and before initiation of chromosome replication (B or G1); chromosome replication (C or S); and cell division (D or G2) $)^{1}$. Since chromosome replication and cell division ( $\mathrm{C}$ and $\mathrm{D}$ periods) remain constant over a wide range of growth rates ${ }^{2,3}$, the step committing cells to initiate chromosome replication largely determines bacterial proliferation rates. Bacteria like Escherichia coli or Bacillus subtilis can increase their growth rate by bypassing the $\mathrm{B}$ period and by initiating replication multiple times per division cycle ${ }^{2}$. In contrast, Caulobacter crescentus strictly separates its cell cycle stages. An asymmetric division generates a sessile stalked (ST) cell, which directly reenters S-phase, and a motile swarmer (SW) cell that remains in G1 for a variable time depending on nutrient availability ${ }^{4,5}$. Coincident with G1/S transition, motile SW cells undergo morphogenesis to gain sessility (Fig. 1a). But what determines the length of G1 in this organism has remained unclear.

In C. crescentus, replication initiation is regulated by the cell cycle kinase CckA. CckA is a bifunctional enzyme that acts as a kinase for the replication initiation inhibitor CtrA in G1, but switches to being a phosphatase in $\mathrm{S}$ phase, resulting in the inactivation of CtrA and clearance of the replication block ${ }^{6}$ (Fig. 1a). The CckA switch is governed by two response regulators, DivK and PleD. While DivK controls CckA activity through protein-protein interactions ${ }^{7-9}, \mathrm{PleD}$ is a diguanylate cyclase, which is responsible for the characteristic oscillation of cdi-GMP during the cell cycle ${ }^{10}$. The concentration of c-di-GMP, below the detection limit in G1, increases during G1/S to reach peak levels at the onset of S-phase ${ }^{11}$ where it allosterically stimulates CckA phosphatase ${ }^{9}$ (Fig. 1a).

Activation of DivK and PleD is directed by the SpmX scaffolding protein, which accumulates during $\mathrm{G} 1 / \mathrm{S}$ and recruits DivJ, the kinase of DivK and PleD, to the incipient stalked cell pole (Fig. 1a) ${ }^{12}$. This makes SpmX accumulation the earliest known event to trigger S-phase entry. However, it is unclear how spmX expression is timed during G1/S to initiate replication. Transcription of spmX is regulated by the response regulator TacA, which in its phosphorylated form also induces the expression of a large set of genes required for SW-to-ST cell morphogenesis ${ }^{12,13}$. TacA is activated via a multistep phosphorylation cascade (Fig. 1b) consisting of the sensor histidine kinase ShkA, and the phosphotransferase protein ShpA ${ }^{12,13}$. ShkA is a multidomain protein kinase with a catalytic domain (CA) that binds ATP and transfers a phosphate via the conserved His residue of the dimerization histidine-phosphotransfer domain (DHp) to a conserved Asp residue of the C-terminal receiver domain (REC2) $)^{12,13}$ (Fig. 1b). TacA controls a large set of target genes including staR, which encodes a transcription factor important for stalk elongation. Thus, this phosphorelay system coordinates replication initiation with morphogenesis. In accordance, cells lacking TacA are elongated, stalk-less and show altered DNA profiles compared with wild type ${ }^{13,14}$. However, the signal activating ShkA has remained unknown (Fig. 1b).

Here we demonstrate that an upshift in levels of the second messenger c-di-GMP determines precise gene expression during G1/S. A combination of genetic, biochemical and cell biology data revealed that c-di-GMP controls the ShkA-TacA pathway by directly binding to the ShkA sensor histidine kinase and strongly stimulating its kinase activity. We demonstrate that binding of cdi-GMP to a pseudo-receiver domain of ShkA abrogates ShkA auto-inhibition leading to the activation of the ShkA-TacA pathway. C-di-GMP-mediated activation of ShkA and the subsequent c-di-GMP-mediated proteolysis of ShkA together define a window of ShkA activity during the cell cycle. This window is sharpened to a narrow, G1/S-specific period by TacA degradation, which precedes the degradation of ShkA. Thus, the exact timing of G1/S-specific gene expression results from the consecutive c-di-GMP-mediated activation and degradation of ShkATacA phosphorylation components.

\section{Results}

C-di-GMP stimulates ShkA kinase activity in vivo and in vitro. A C. crescentus strain lacking all diguanylate cyclases $\left(\mathrm{cdG}^{0}\right.$ strain), which was generated to remove all traces of the second messenger c-di-GMP, shows strong developmental and morphological abnormalities with mutant cells being irregularly shaped, elongated and lacking all polar appendages ${ }^{11}$. Because stalk biogenesis depends on an active ShkA-TacA phosphorelay (Fig. 1b) ${ }^{13}$, we tested if c-di-GMP controls the ShkA-TacA pathway. Expression of TacA ${ }^{\mathrm{D} 54 \mathrm{E}}$, a phospho-mimetic form of $\mathrm{TacA}^{13}$, restored stalk biogenesis, transcription of the TacA targets staR and spmX, as well as localization of a SpmX-mCherry fusion to the stalked pole (Fig. 1c, d; Supplementary Fig. 1). spmX and $s t a R$ transcription was also restored when c-di-GMP was reintroduced by expression of the heterologous diguanylate cyclase (DGC) $d g c Z$ from $E$. coli in the $\mathrm{cdG}^{0}$ background or in a strain that lacks all DGCs and phosphodiesterases (PDE) ( $\mathrm{rcdG}^{0}$ strain) (Fig. 1d; Supplementary Fig. 1). Phos-tag PAGE analysis revealed that TacA and ShkA were unphosphorylated in the $\mathrm{cdG}^{0}$ strain. This phosphorylation was restored upon expression of the constitutively active DGC PleD ${ }^{\star 15}$ (Fig. 1e). In contrast, expression of the heterologous phosphodiesterase PA5295 from Pseudomonas aeruginosa, which also lowers c-di-GMP levels, in wildtype cells reduced TacA and ShkA phosphorylation (Fig. 1f). Stalk formation is independent of the ShkA-TacA pathway when cells are grown in a low-phosphate medium ${ }^{9}$. In agreement with this, a strain lacking c-di-GMP formed stalks at low-phosphate concentrations, but failed to localize DivJ (Supplementary Fig. 1). Many cells displayed bipolar stalks, indicating that at lowphosphate c-di-GMP controls stalk positioning through a pathway distinct from ShkA-TacA. Altogether, these results indicate that c-di-GMP acts upstream of and is required for the activity of the ShkA-TacA phosphorelay.

In vitro phosphorylation assays with purified ShkA or with all components of the phosphorelay demonstrated that c-di-GMP strongly and specifically stimulates ShkA autokinase activity (Fig. 1g, h; Supplementary Fig. 2a). Moreover, purified ShkA bound c-di-GMP with a $K_{D}$ in the sub-micromolar range (Supplementary Fig. 2b). Altogether, we conclude that c-diGMP activates the ShkA-TacA pathway by directly binding to and allosterically stimulating ShkA kinase.

C-di-GMP activates ShkA by binding to its pseudo-receiver domain. We next sought to dissect the mechanism of c-di-GMPmediated ShkA activation. We devised a genetic selection (see "Methods") to isolate shkA mutations that restored spmX expression in a $\mathrm{rcdG}^{0}$ background. Independent mutations were identified in two residues (D369, R371) within a short stretch of three highly conserved amino acids in the linker region between REC1 and REC2 (hereafter referred to as the "DDR" motif) (Fig. 2a; Supplementary Fig. 3). These results identified the REC1REC2 linker region as a critical determinant of ShkA activation.

We further characterized the D369N variant both in a wild-type background and in a strain expressing a stabilized version of TacA $\left(\mathrm{Tac} \mathrm{A}^{\mathrm{DD}}\right)$, and confirmed that TacA phosphorylation levels and spmX transcription were restored to wild-type levels in the $\mathrm{rcdG}^{0}$ background harboring the $\operatorname{sh} k A^{D 369 N}$ allele (Fig. 2b, c). Also, purified ShkA ${ }^{\mathrm{D} 369 \mathrm{~N}}$ protein showed strong autophosphorylation activity even in the absence of c-di-GMP (Fig. 2d). The mutant retained its ability to bind c-di-GMP in vitro (Supplementary Fig. 2c) and could still be partially stimulated by c-di-GMP 
a

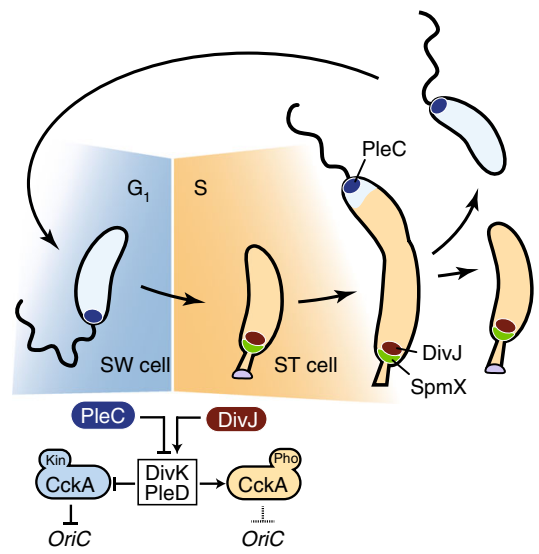

b

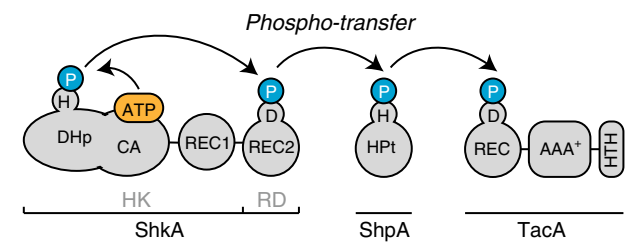

d

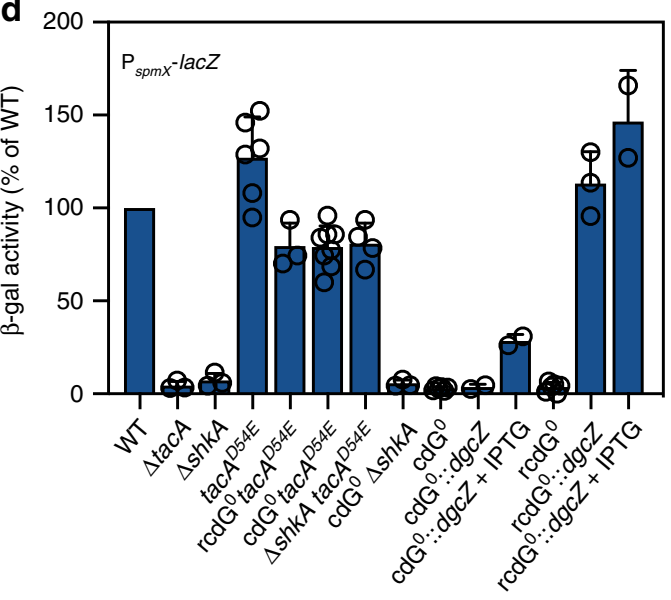

g

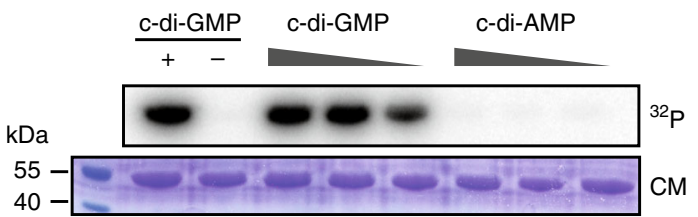

c

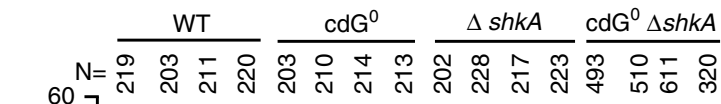

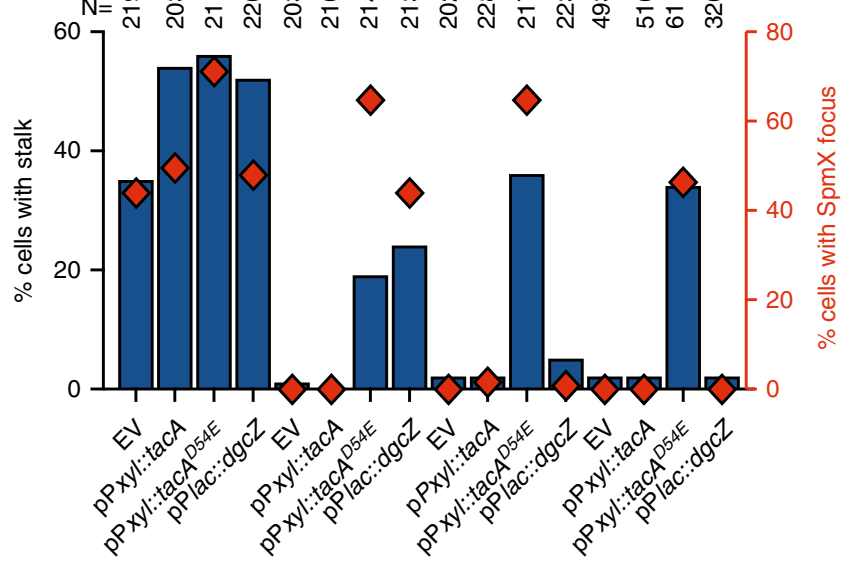

e

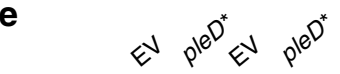

f

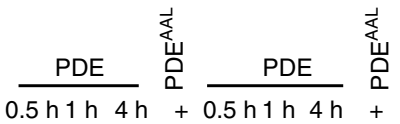

ShkA P ShkA -

$\frac{1.03 .4}{\mathrm{WT}} \frac{0.33 .4}{\mathrm{cdG}^{0}}$

TacA P

TacA $=---$

Rel. phos. $\frac{1.01 .5}{W T} \frac{0.11 .7}{\mathrm{cdG}^{0}}$

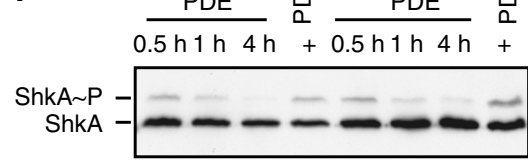

Rel. phos. $\begin{array}{llllllll}0.8 & 0.5 & 0.2 & 1.0 & 0.7 & 0.2 & 0.2 & 1.0\end{array}$ ShkA ShkA ${ }^{\mathrm{DD}}$

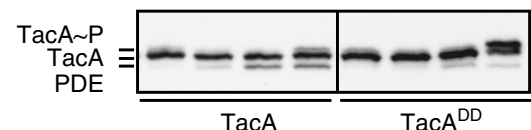

h

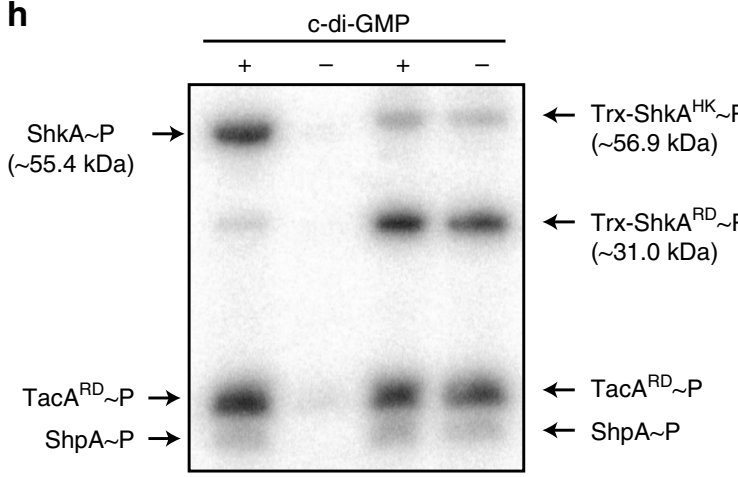

Fig. 1 C-di-GMP controls the ShkA-TacA phosphorelay in C. crescentus. a Schematic of the $C$. crescentus cell cycle with swarmer and stalked cells colored in blue and orange, respectively, and the G1- and S-phases of the cell cycle indicated in similar colors. Stage-specific kinase (Kin) and phosphatase (Pho) activities of CckA are indicated with the coloring referring to stage-related activities. DivK and PleD that control the CckA switch are highlighted. b Schematic of the ShkA-ShpA-TacA phosphorelay. c Quantification of cells with stalks and SpmX-mCherry foci of strains expressing a chromosomal spmX-mCherry fusion and plasmid-driven tacA, tac $A^{D 54 E}$, or dgcZ. EV, empty vector. The number of cells analyzed $(N)$ is indicated above the graph. Also see Supplementary Fig. 1. d spmX promoter activity (plasmid pRKlac290-spmX) in indicated strains. IPTG indicates induction of dgcZ expression. Shown are mean values and standard deviations $(N \geq 2)$. e Phos-tag PAGE immunoblots of strains producing 3xFLAG-tagged ShkA or TacA from the native loci and expressing plasmid-borne PleD*. Relative phosphorylation (rel. phos.) of TacA and ShkA were determined by calculating the ratio of intensities of phosphorylated and unphosphorylated bands normalized to this value of the wild type with empty vector control. $\mathbf{f}$ Phos-tag PAGE immunoblots of strains encoding 3xFLAG-tagged ShkA or TacA at the native loci or the respective mutant alleles encoding degradation-resistant variants TacADD or ShkADD. Strains carried a plasmid for cumate-inducible expression of wild type or a catalytic mutant (AAL) of the phosphodiesterase (PDE) PA5295. Samples were harvested after addition cumate as indicated. PA5295 $\mathrm{AAL}$ samples were harvested $4 \mathrm{~h}$ post induction. Relative phosphorylation (rel. phos.) was normalized to the PA5295 AAL control. $\mathbf{g}$ In vitro phosphorylation of purified ShkA with different nucleotides present $(1 \mathrm{mM}, 100 \mu \mathrm{M}, 10 \mu \mathrm{M})$. Positive $(+)$ and negative (-) controls contained $100 \mu \mathrm{M}$ c-di-GMP and $\mathrm{ddH}_{2} \mathrm{O}$, respectively. Reactions were initiated by addition of $500 \mu \mathrm{M}$ radiolabeled ATP and allowed to proceed for $15 \mathrm{~min}$ at room temperature. $\mathbf{h}$ In vitro phosphorylation assays with purified components ( $4 \mu \mathrm{M})$ of the ShkA-ShpA-TacA phosphorelay with $(+)$ or without $(-)$ c-di-GMP $(76 \mu \mathrm{M})$. Reactions were initiated as described for $\mathbf{g}$. Source data are provided as a Source Data file. 
a

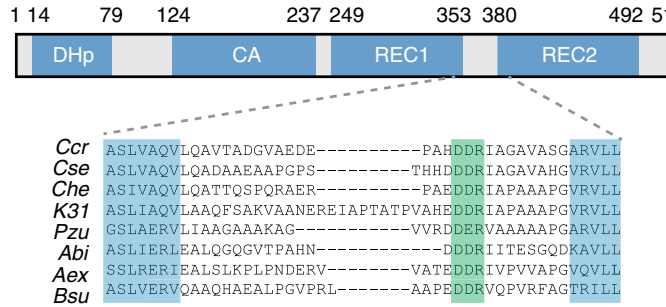

d

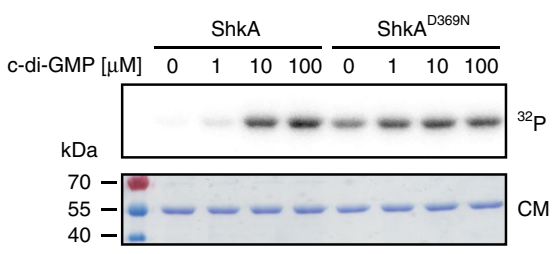

f

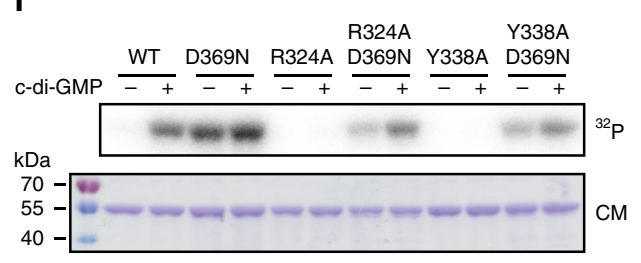

g b

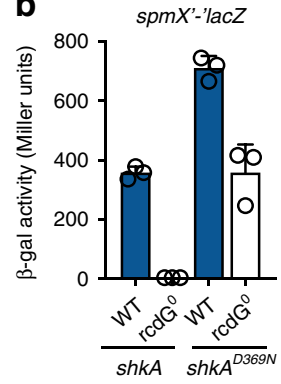

C

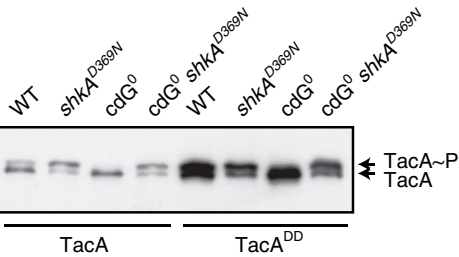

e

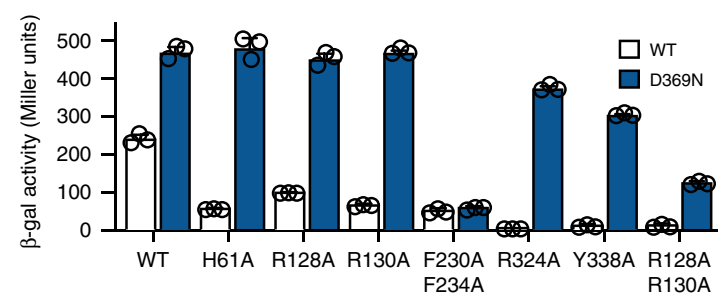

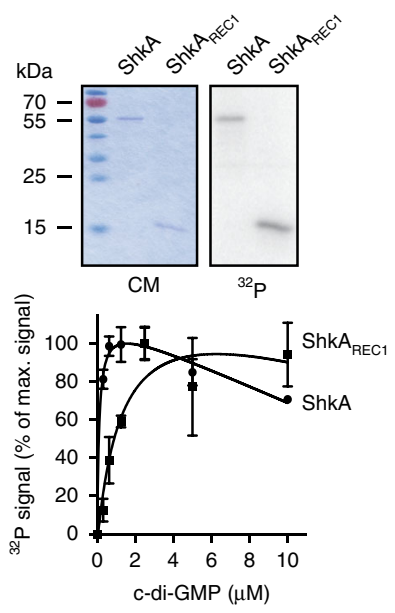

j

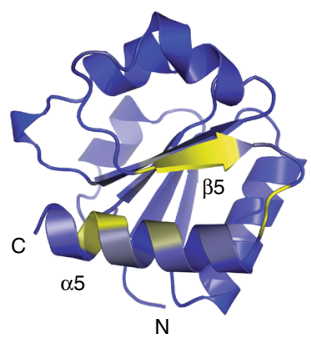

$\mathbf{k}$

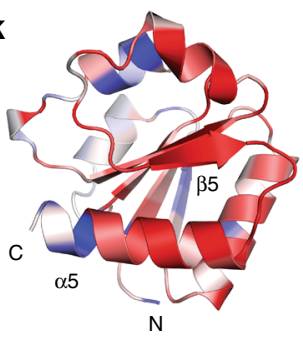

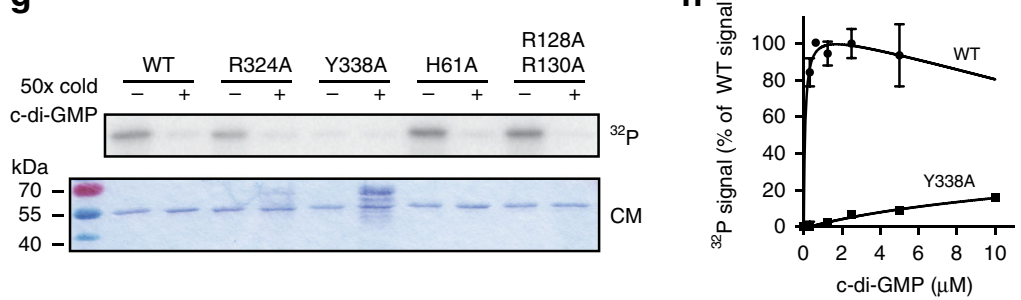

both in vivo (Fig. 2b) and in vitro (Supplementary Fig. 2d). Thus, mutations in the DDR motif uncouple ShkA activity from c-di-GMP without interfering with c-di-GMP binding.

We used the c-di-GMP independence of the D369N ShkA variant to identify residues involved in c-di-GMP binding. We reasoned that mutations in ShkA specifically interfering with cdi-GMP-mediated activation should be rescued when combined with D369N. In contrast, mutants causing more general kinase defects (ATP-binding, phosphotransfer, protein stability etc.) would not be recuperated when combined with D369N. An alignment of ShkA orthologs from C. crescentus and related organisms revealed a total of 25 candidate residues for c-di-GMP binding distributed throughout the entire protein (Supplementary Fig. 3). Of all the mutants that severely affected ShkA activity
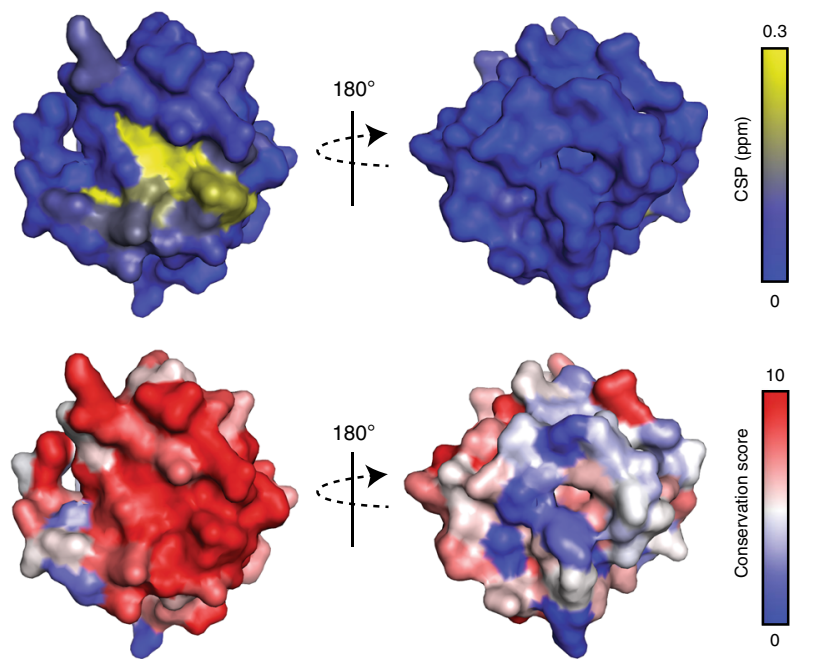

(Supplementary Fig. 4a, b) and that were rescued in vivo (Fig. 2e) and in vitro (Fig. 2f) when combined with D369N, only one, Y338A, in REC1, interfered with c-di-GMP binding (Fig. 2g, h), while the other mutations likely affect c-di-GMP-mediated conformational changes required for activation.

Thus, Y338 in REC1 is likely a part of the c-di-GMP binding site. Indeed, the purified REC1 domain alone was able to bind cdi-GMP, although with lower affinity than full-length ShkA (Fig. 2i). NMR spectroscopy with REC1 revealed a fold reminiscent of prototypical REC domains, except that helix $\alpha 3$ is not present irrespective of the presence or absence of c-di-GMP (Supplementary Fig. 4c-f). Upon the addition of c-di-GMP, REC1 shows chemical shift perturbations (CSPs), which cluster on the $\beta 5-\alpha 5$ surface (Fig. 2j; Supplementary Fig. 4g). Importantly, 
Fig. 2 C-di-GMP binds to the REC1 pseudo-receiver domain. a ShkA domain architecture drawn to scale (top) and alignment of the REC1-REC2 linker harboring the DDR motif (highlighted in green) of ShkA orthologs (bottom). Ccr, C. crescentus; Cse, Caulobacter segnis; Che, Caulobacter henricii; K31, Caulobacter sp. K31; Pzu, Phenylobacterium zucineum; Abi, Asticcacaulis biprosthecium; Aex, Asticcaucaulis excentricus; Bsu, Brevundimonas subvibrioides. Dimerization and histidine-phosphotransfer (DHp), catalytic and ATP-binding (CA) and receiver (REC1, REC2) domains are indicated. b Activity of the spmX promoter in indicated strains harboring plasmid pAK502-spmX. shkA ${ }^{D 69 N}$ is expressed from the native chromosomal locus. Means and standard deviations are shown $(N=3)$. c Phos-tag PAGE immunoblots of indicated strains producing $3 \times F L A G$-tagged TacA or TacADD from the native locus. $\mathbf{d}$ In vitro autophosphorylation assays of ShkA and ShkAD369N. Top: autoradiograph; bottom: Coomassie stain. e $\beta$-Gal assays of $\Delta$ shkA mutant strains harboring plasmid pAK502-spmX and expressing different shkA alleles in trans from plasmid pQF with the indicated amino acid substitutions alone (WT, white bars) or in combination with D369N (blue bars). Shown are means and standard deviations $(N=3)$. $\mathbf{f}$ In vitro autophosphorylation assays of wild type or mutant ShkA with $(10 \mu \mathrm{M})$ or without c-di-GMP. Reactions were run for $5 \mathrm{~min}$. Top: autoradiograph; bottom: Coomassie stain. $\mathbf{g}$ Autoradiographs of purified ShkA and ShkA mutant variants $(0.5 \mu \mathrm{M})$ UV-crosslinked with $10 \mu \mathrm{M}$ [32P]c-di-GMP with or without addition of a 50 -fold molar excess of nonlabeled c-di-GMP. Top: autoradiograph; bottom: Coomassie stain. $\mathbf{h}$ Quantified autoradiographs of purified ShkA and ShkA $3338 \mathrm{~A}$ (0.5 $\mu$ M) UV-crosslinked with increasing concentrations of [32 P]c-di-GMP. Shown are mean values and standard deviations $(N=2)$. i Autoradiographs and Coomassie stain of the same gel of purified ShkA and the isolated REC1 domain (ShkA $\left.A_{R E C 1}\right)(0.5 \mu \mathrm{M})$ after UV crosslinking with $10 \mu M$ [ $\left.{ }^{32} \mathrm{P}\right] \mathrm{c}$-di-GMP (top). Quantified autoradiographs of purified ShkA and ShkA $A_{R E C 1}(0.5 \mu M)$ after UV crosslinking with increasing concentrations of [ $\left.{ }^{32} \mathrm{P}\right] \mathrm{c}$-di-GMP (bottom). Means and standard deviations are shown $(N=2)$. $\mathbf{j}$ Cartoon and surface representation of the ShkA $A_{R E C 1}$ homology model with NMR chemical shift perturbations (CSPs) upon c-di-GMP binding indicated by a blue-to-yellow gradient. $\mathbf{k}$ Conservation score of ShkA orthologs (see "Methods"). Source data are provided as a Source Data file.

these residues include Y338 and are well conserved (Fig. 2k; Supplementary Fig. 3). Some of the other residues implicated in cdi-GMP binding by NMR were also important for ShkA activity in vivo (Supplementary Fig. 4h). Altogether, these experiments revealed a REC1 pseudo-receiver domain of ShkA as the primary docking site for c-di-GMP, and identified residues directly involved in c-di-GMP binding and c-di-GMP-mediated activation of ShkA. In silico analysis revealed that pseudo-receiver domains, although often not annotated, are widespread among histidine kinases (Supplementary Fig. 5, Supplementary Data 1). We propose that pseudo-receiver domains have lost their original phosphotransfer function but during evolution have adopted signal perception functions and may thus represent a large class of so-far unrecognized kinase input domains.

Similar to the two identified DDR mutants, the kinase activity of ShkA is also uncoupled from c-di-GMP when conserved residues of REC2 are mutated (Supplementary Fig. 2e) or when the C-terminal REC2 domain is removed from the catalytic core (DHp, CA, and REC1 domain) (Fig. 1h). Thus, the REC2 domain and the DDR linker motif inhibit ShkA autophosphorylation, maintaining it in an inactive state. We propose that c-di-GMP binding to REC1 overrides this auto-inhibition and activates the enzyme.

\section{C-di-GMP defines a narrow window of ShkA activity during} G1/S. The c-di-GMP-independent variants of ShkA allowed us to more carefully investigate the role of ShkA in the temporal control of events during G1/S. Introduction of the $\operatorname{shk} A^{D 369 N}$ allele into the $\mathrm{rdG}^{0}$ strain restored SpmX protein levels, stalk biogenesis, DivJ localization to the incipient stalked pole, and normal cell morphology (Fig. 3a, b; Supplementary Fig. 6a). Of note, shk $A^{D 369 N}$ failed to restore G2-specific processes in the $\mathrm{rcdG}^{0}$ strain like motility and type IV pili assembly (Supplementary Fig. 6b, c). Restoration of cell morphology and DivJ localization was entirely dependent on SpmX (Fig. 3b), arguing that c-di-GMP and ShkA contribute to cell cycle progression and morphogenesis via spmX expression control. Consistent with this notion, the $\operatorname{sh} k A^{D 369 N}$ allele rescued the synthetic-lethal phenotype of a strain lacking c-di-GMP and suffering from reduced DivK levels 9 in a SpmX-dependent manner (Supplementary Fig. 6), indicating that ShkA influences the CckA kinase/phosphatase balance. A strain harboring the $\operatorname{sh} k A^{D 369 N}$ allele prematurely produced SpmX already in newborn G1 cells (Fig. 3c). Accordingly, the shk $A^{D 369 N}$ mutant exited G1 prematurely, as indicated by the strong reduction of G1 cells (Fig. 3d). Likewise, cells failed to properly arrest in G1 after entry into the stationary phase (Fig. 3d, e). Growth rate of the $\operatorname{sh} k A^{D 369 N}$ mutant was not increased in either PYE or M2G medium compared with wild type, suggesting that the overall length of the cell cycle (combined G1, S, and G2 phases) is not or only marginally affected (Supplementary Fig. 6). These results support a role of c-di-GMP, and the ShkA-TacA pathway, in the timely execution of the G1/S transition.

TacA was previously shown to be degraded by the ClpXP protease, a process that requires the adapter proteins $\mathrm{RcdA}$ and CpdR and depends on the C-terminal Ala-Gly degradation motif of TacA ${ }^{16}$. ShkA harbors the same C-terminal degradation signal and was also degraded upon S-phase entry about $10 \mathrm{~min}$ after the removal of TacA (Fig. 3f). When the C-terminal Ala-Gly motif was replaced by Asp residues (ShkA $\left.{ }^{D D}\right)$, ShkA and ShkA P were stabilized and prevailed throughout the cell cycle (Fig. 3f). Sequential degradation of TacA and ShkA may be explained by their differential requirements for protease adapters. While TacA degradation by ClpXP depends on CpdR and RcdA ${ }^{16}$, ShkA degradation also requires PopA, the third member of the adapter hierarchy regulating $\mathrm{ClpXP}$ protease activity during the $C$. crescentus cell cycle (Fig. $3 g, h$ ). Because PopA needs to bind cdi-GMP to act as a protease adapter ${ }^{17}$, the ShkA-TacA pathway is confined to G1/S by the sequential c-di-GMP-dependent activation and c-di-GMP-mediated degradation of the ShkA kinase.

The ShkA-TacA pathway limits gene expression to G1/S. To carefully assess the contribution of ShkA activation and degradation for the temporal control of $s p m X$, the $\operatorname{spmX}$ promoter was fused to the fluorescent protein Dendra2. The photoconvertible properties of Dendra $2^{18}$ allowed determining both "ON" and "OFF" kinetics of $\operatorname{spmX}$ promoter activity during the cell cycle (Fig. 4b; Supplementary Fig. 7a). These experiments revealed that spmX promoter activity peaks during G1/S roughly 15-30 min after passing through the predivisional stage (Fig. $4 \mathrm{~b}$ ). The $s p m X$ promoter was active in cells progressing through G1/S, but not in newborn ST progeny that reenter S-phase immediately at the end of the asymmetric cell cycle (Fig. 4a, c; Supplementary Fig. 7a-c). Stage-specific expression of $s p m X$ required a combination of c-diGMP oscillations during the cell cycle and TacA degradation: expression of combinations of $d g c Z$, a gene encoding a highly active DGC from $E$. coli ${ }^{19}$, and of $\operatorname{tacA}^{D D}$ (encoding stable $\operatorname{TacA}^{16}$ ) and $\operatorname{shk} A^{D 369 N}$ (encoding constitutive ShkA) alleles resulted in a gradual loss of G1/S-specific spmX expression 
a
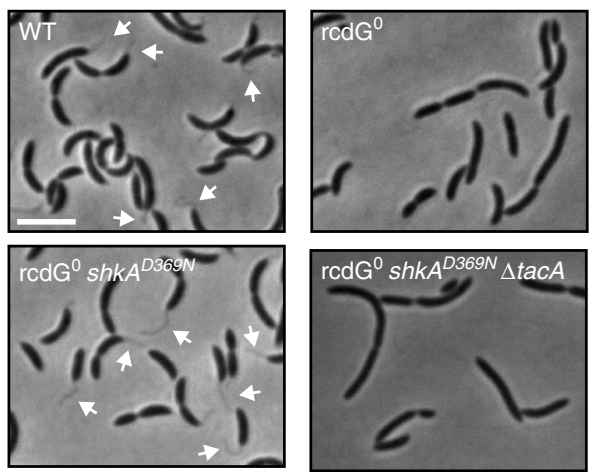

b

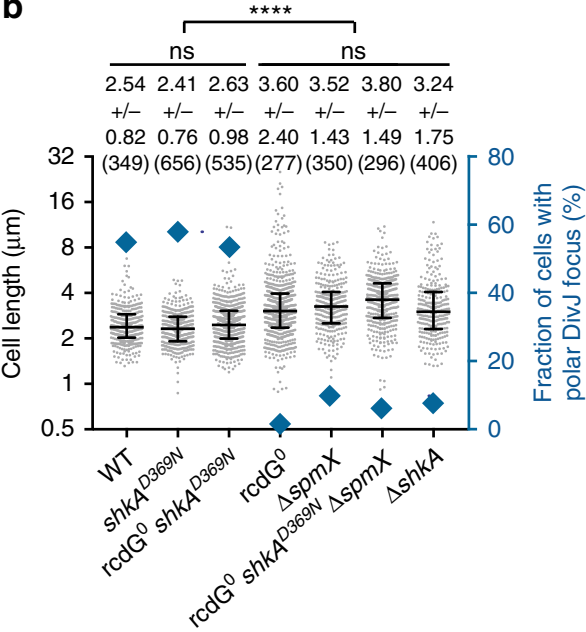

f

\begin{tabular}{l} 
facA \\
\cline { 2 - 3 }
\end{tabular}

WT

DD

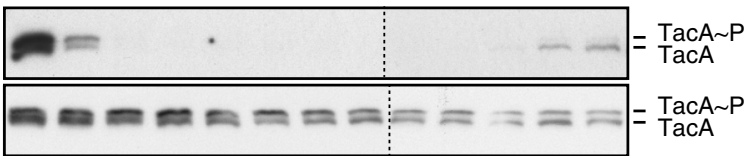

$\underline{\text { ShkA }}$

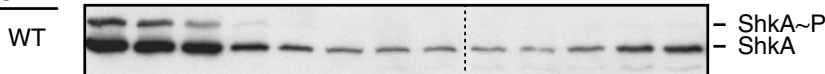

DD

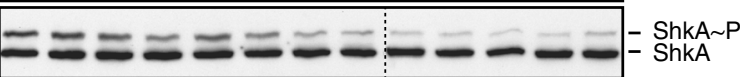

d

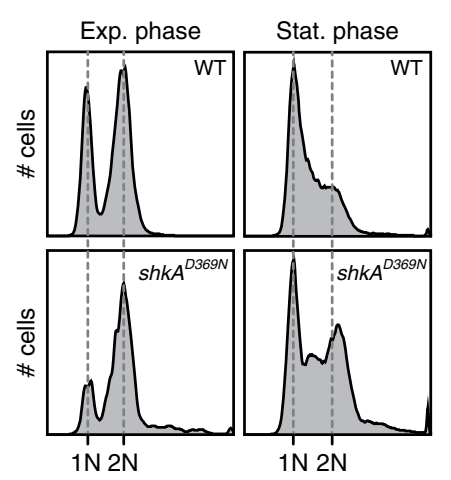

e

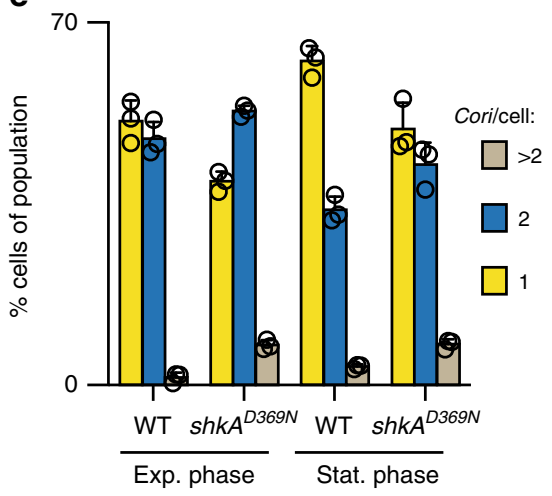

g

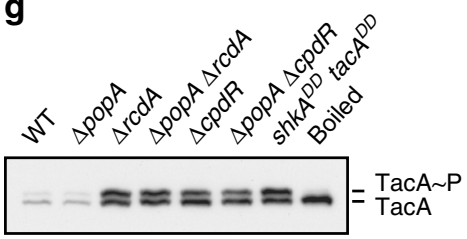

h

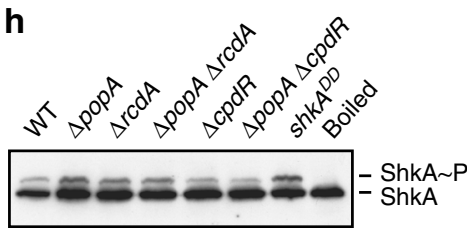

Fig. 3 C-di-GMP imposes precise temporal control of TacA activity during G1/S. a Representative phase-contrast micrographs of indicated strains. Arrows point to stalks. The scale bar represents $4 \mu \mathrm{m}$. b Quantification of cell length and polar DivJ localization (DivJ-mCherry) of indicated strains. Median values with interquartile ranges are shown in the graph and mean values and standard deviations are indicated above the graph. The number of cells analyzed is shown in brackets. ${ }^{\star \star \star \star}$ indicates a $P$ value of $<0.0001$; ns, not significant (ordinary one-way ANOVA and Tukey's multiple comparison test). $\mathbf{c}$ Immunoblots of synchronized cultures of $C$. crescentus strains expressing $3 x F L A G$-shkA or $3 x F L A G$-shkAD369N from the native chromosomal locus were probed with anti-FLAG, anti-TacA, anti-SpmX and anti-CtrA antibodies. $\mathbf{d}$ Analysis of chromosome content by flow cytometry of indicated strains in exponential or stationary phase after rifampicin treatment. e Quantification of chromosome number of indicated strains. Shown are means and standard deviations $(N=3)$. f Phos-tag PAGE immunoblots of synchronized cultures of strains expressing chromosomally encoded 3xFLAG-tacA, 3xFLAG-shkA, 3xFLAG-tacADD, or 3xFLAG-shkADD alleles were probed with anti-FLAG antibody. $\mathbf{g}$ Phos-tag PAGE immunoblots of mixed cultures of indicated mutant strains expressing 3xFLAG-tacA probed with anti-FLAG antibodies. h Phos-tag PAGE immunoblots of mixed cultures of indicated mutant strains expressing 3xFLAG-shkA probed with anti-FLAG antibodies. Source data are provided as a Source Data file.

(Fig. 4c; Supplementary Fig. 7c). These experiments provided direct evidence that the activity of the ShkA-TacA pathway is strictly limited to G1/S and SW progeny that need to passage through the G1 phase of the cell cycle.

To investigate the importance of limiting the ShkA-TacA pathway to G1/S for accurate cell cycle progression, we examined the consequences of ShkA-TacA dysregulation using stable
$\left(\operatorname{TacA}^{\mathrm{DD}}, \quad\right.$ ShkA $\left.{ }^{\mathrm{DD}}\right)$, or constitutively active $\left(\right.$ ShkA $\left.{ }^{\mathrm{D} 369 \mathrm{~N}}\right)$ variants or combinations thereof. All alleles increased overall spmX expression and showed additive effects when combined (Supplementary Fig. 8a). Cell division and cell morphology were normal in all strains that either retained TacA degradation or cdi-GMP-mediated ShkA activity control. However, when mutations that stabilize ShkA or TacA were combined with a 
a

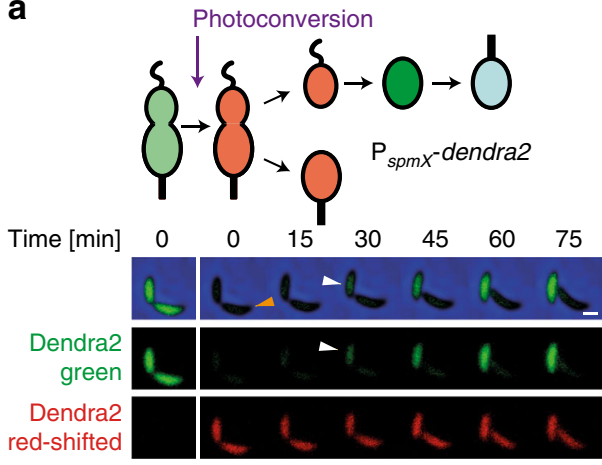

b

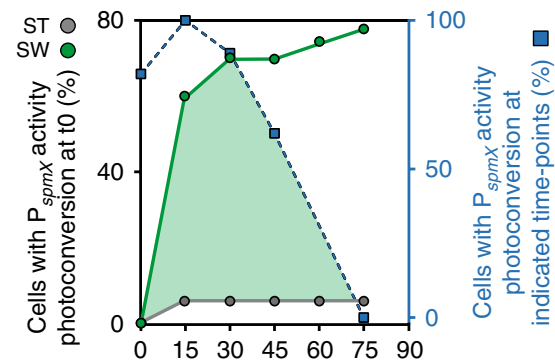

C

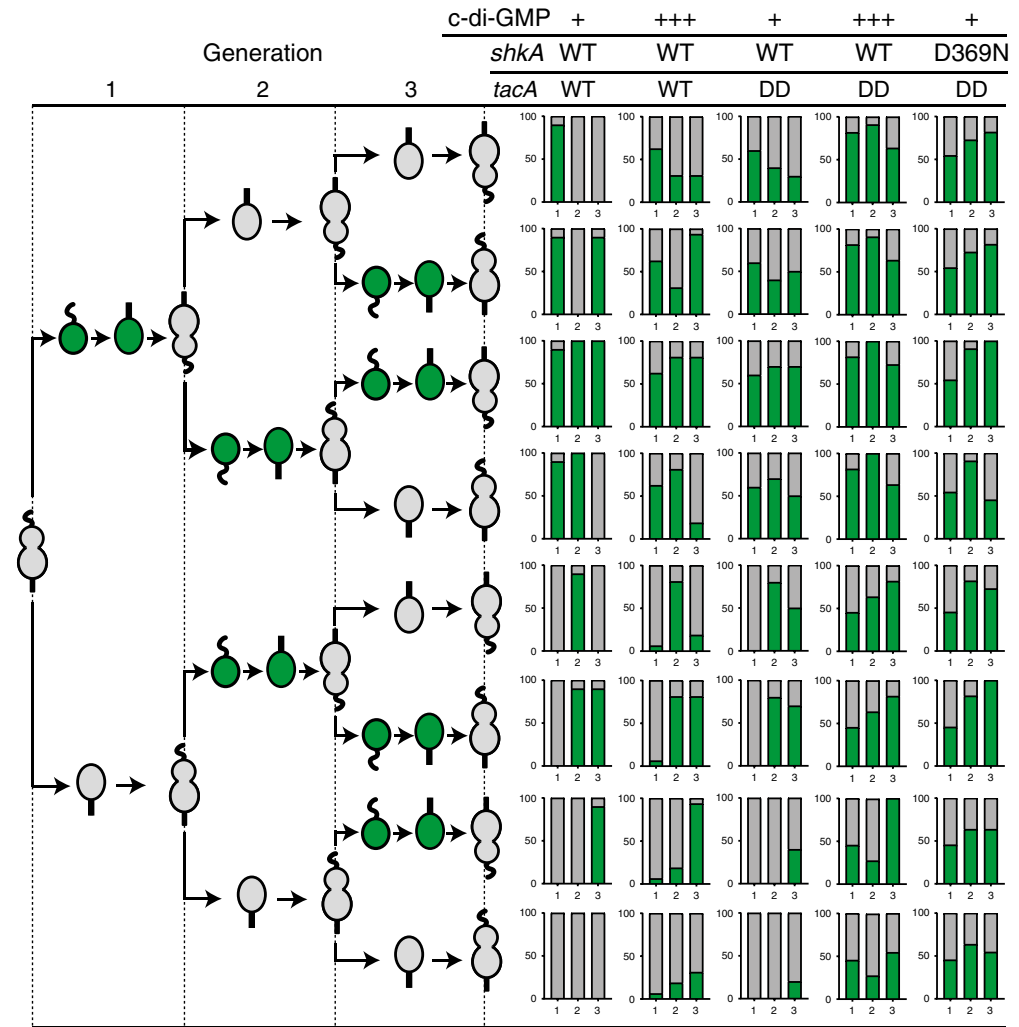

d

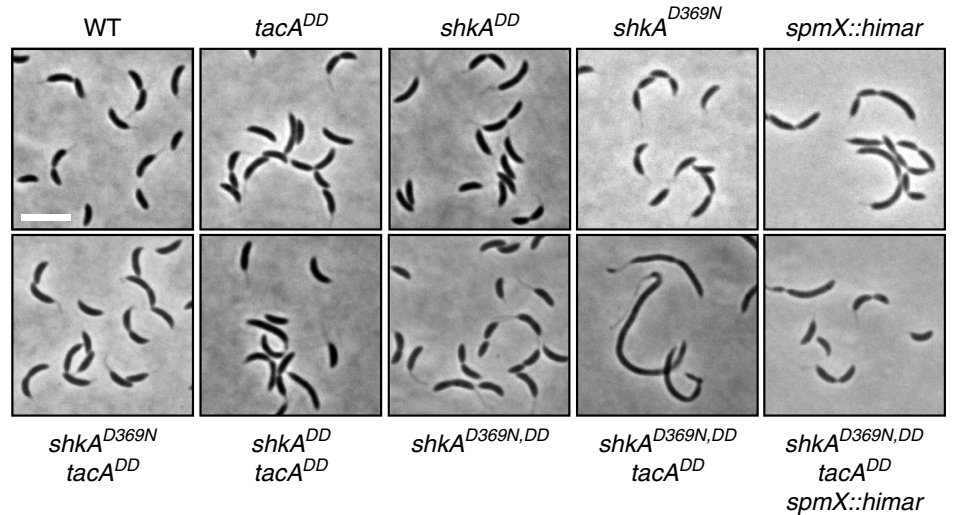

mutation constitutively activating ShkA, strains showed strong cell division and morphology aberrations, effects that were strictly dependent on an intact copy of $\operatorname{spmX}$ (Fig. $4 \mathrm{~d}$; Supplementary Fig. 8b). Thus, dysregulation of the SpmX morphogen leads to aberrant cell morphogenesis. Together, these experiments provide evidence that dysregulation of ShkATacA activity leads to severe cell cycle and morphological defects.
The diguanylate cyclase PleD activates ShkA during G1/S. The above data support a model in which an upshift of c-di-GMP stimulates ShkA kinase activity thereby initiating the G1/S-specific genetic program (Fig. 5a). If so, the G1/S transition should be kick-started by one of the $C$. crescentus diguanylate cyclases. Screening a spmX-lacZ reporter strain for transposon insertions with reduced lac $Z$ expression identified mutations in pleD. 
Fig. 4 Oscillation of c-di-GMP and protein degradation limit TacA activity to G1/S. a Time-lapse fluorescence microscopy of C. crescentus wild-type cells expressing dendra2 from the spmX promoter. The time of photoconversion of Dendra2 (green to red) in the predivisional cell (PD) is indicated in the schematic of the $C$. crescentus cell cycle. A representative example of a dividing cell is shown below with separate green and red channels and with the ST cell pole of the PD cell marked (orange arrow). spmX expression (green) is restricted to the SW cell during G1/S transition (white arrows). The bar is $1 \mu \mathrm{m}$. b ON and OFF kinetics of $\operatorname{spmX}$ promoter activity during G1/S in wild-type cells harboring the spmX-dendra2 reporter plasmid. ON kinetics were determined as outlined in a with cells being photoconverted before division ( $0 \mathrm{~min}$ ) and the fraction of ST (gray circles) and SW cells (green circles) with induced green fluorescence plotted over time. OFF kinetics were determined by the fraction of SW cells with induced green fluorescence after photoconversion at the indicated time points during the cell cycle (blue boxes). The overlap of the two curves (green area) defines the window of spmX promoter activity during the cell cycle. c Activity of spmX promoter in lineages of individual C. crescentus cells of different strains through three consecutive generations as indicated by the schematic on the left. The projected G1/S-specific expression of spmX is indicated in green. For each strain 10-16 late PD harboring the spmX-dendra2 reporter were photoconverted roughly $15 \mathrm{~min}$ before cell division and followed by time-lapse microscopy through three cell division events. Right: The fraction of SW and ST offspring (\% of total cells analyzed) with active spmX promoter (green) was plotted over three generations with the $x$-axis representing generations 1-3. For experimental details and data analysis see "Methods". C-di-GMP levels were manipulated by Plac-driven dgcZ with 0.1 mM IPTG. EV, empty vector control. d Representative phase-contrast micrographs of strains carrying different shkA and tac $A$ alleles encoding stabilized $\left(s h k A^{D D}\right.$ and $t a c A^{D D}$ ) or c-di-GMP-independent (shkAD369N) versions of the respective proteins. The scale bar represents $4 \mu \mathrm{m}$.

Accordingly, a $\Delta p l e D$ deletion strain showed strongly reduced spmX expression (Fig. 5b). While a second diguanylate cyclase enzyme, DgcB, had a more modest effect, spmX promoter activity was almost completely abolished in a $\Delta p l e D \quad \Delta d g c B$ double mutant, akin to a strain lacking c-di-GMP (Fig. 5b). Thus, PleD is the major diguanylate cyclase driving the G1/S-specific transcriptional program.

PleD phosphorylation is regulated by the antagonistic ST cellspecific kinase DivJ and the SW cell-specific phosphatase PleC (Figs. 1a and 5a) ${ }^{8}$. However, PleC but not DivJ, was required for spmX expression, and spmX expression was restored to normal levels in a strain lacking both $\mathrm{PleC}$ and DivJ (Fig. 5b). This indicated that neither DivJ nor $\mathrm{PleC}$ is responsible for the initial activation of PleD and for ShkA stimulation, and that the role of $\mathrm{PleC}$ is likely indirect. $\mathrm{PleC}$ phosphatase was previously shown to reduce DivK phosphorylation leading to the activation of CtrA. Because tacA is a direct target of CtrA, it was proposed that in a $\triangle p l e C$ mutant, spmX expression is impaired because TacA fails to accumulate $^{12}$. Expression of $t a c A$ and spmX indeed required the PleC phosphatase (Fig. $5 c$, d). However, when the pleC deletion was combined with the constitutive shkA ${ }^{D 369 N}$ allele, SpmX protein levels and polar localization of DivJ, but not tacA expression, were fully restored (Fig. 5c, e; Supplementary Fig. 9), arguing that TacA levels are not the limiting factor in cells lacking PleC. Rather, ShkA activity and its stimulation by c-di-GMP are switched off in the $\triangle p l e C$ mutant. Indeed, a constitutively active variant of PleD, PleD ${ }^{\star 15}$, restored SpmX protein levels in the $\Delta p l e C$ mutant, an effect that was entirely dependent on ShkA (Fig. 5f). These results suggest that c-di-GMP levels are limiting in a $\Delta p l e C$ mutant. In line with this, LC-MS measurements showed that the $\triangle p l e C$ mutant has significantly reduced c-di-GMP levels as compared with the isogenic ple $C^{+}$strain $(9.5 \pm 1.3 \mu \mathrm{M}$ vs. $16.3 \pm 0.5 \mu \mathrm{M} ; N=3$ ).

The finding that the ShkA-TacA pathway is OFF in the $\Delta p l e C$ mutant because c-di-GMP concentrations are limiting, together with the observation that PleD serves as the main c-di-GMP donor for ShkA activation, argues for the existence of an as yet unidentified PleD kinase, the expression of which will likely depend on the PleC-CckA-CtrA cascade. We speculate that activation of this kinase and its downstream target $\mathrm{PleD}$ represents a key event in the decision of $C$. crescentus to exit G1 (Fig. 5a).

\section{Discussion}

C. crescentus SW cells are born with low levels of c-di-GMP11,20. This is imposed by two cell type-specific regulators, the phosphodiesterases $\mathrm{Pde}^{21}$ and the phosphatase $\mathrm{PleC}$, which maintains the diguanylate cyclase PleD in its inactive, unphosphorylated form $^{8}$ (Fig. 5a). During the G1/S transition, PdeA is proteolytically removed ${ }^{21}$ and PleD is activated by phosphorylation. This results in a gradual increase of $\mathrm{c}$-di-GMP ${ }^{11,20}$, which leads to a series of accurately timed events prompting exit from G1, cell morphogenesis and entry into S-phase. First, ShkA is allosterically activated resulting in TacA phosphorylation and the expression of a large group of G1/S-specific genes that orchestrate the morphological restructuring of the motile SW cells into sessile ST cells ${ }^{13,14}$. At this stage, c-di-GMP levels are high enough to activate ShkATacA but have not reached the peak levels needed to trigger the CckA cell cycle switch and S-phase entry ${ }^{8,9}$. This leads to the execution of the morphogenetic program before cells commit to chromosome replication and division. The next step is then catalyzed by the expression of one of the G1/S-specific proteins, the morphogen SpmX, which is responsible for the polar sequestration and activation of Divj ${ }^{12}$. This, in turn, leads to the production of more c-di-GMP via reinforced activation of $\mathrm{PleD}$ and, together with DivJ-mediated phosphorylation of DivK, switches CckA into a phosphatase and ultimately licenses replication initiation ${ }^{7-9}$ (Fig. 5a). ShkA binds c-di-GMP with five- to tenfold higher affinity than CckA $^{9,17}$ explaining how the ShkA-TacA pathway and the CckA switch can be sequentially activated. Thus, at least four kinases form a hierarchical cascade $(\mathrm{HK} \rightarrow \mathrm{ShkA} \rightarrow$ DivJ $\rightarrow$ CckA) that is responsible for the accurate temporal control of events during G1/S. The activity and timing of this cascade is coordinated by the second messenger c-di-GMP, a stepwise increase of which enforces consecutive cell cycle steps by modulating the activity of ShkA and CckA, respectively (Fig. 5a).

By contributing to the CckA phosphatase switch via SpmX and DivJ, the ShkA-TacA pathway initiates its own termination. The CckA phosphatase activates a protease adapter cascade that includes CpdR and PopA ${ }^{16}$ and that leads to the consecutive degradation of TacA and ShkA by the ClpXP protease (Fig. 5a). This negative feedback constitutes an intrinsic, self-sustained timer that shuts down ShkA-TacA activity as soon as c-di-GMP has reached peak levels required to activate the CckA phosphatase and the PopA protease adapter, thereby irreversibly committing cells to S-phase. Because TacA not only controls genes involved in cell cycle progression but also regulates morphological restructuring of the motile SW cells into sessile ST cells ${ }^{13,14}$, accurate temporal control of this pathway secures the tight coordination between replicative and behavioral processes.

Our results show that the diguanylate cyclase PleD is largely responsible for the c-di-GMP upshift during G1-S transition. We postulate that the initial event leading to PleD activation during G1/S must be executed by a kinase other than DivJ or PleC, and that DivJ is part of a positive feedback loop that reinforces PleD 
a

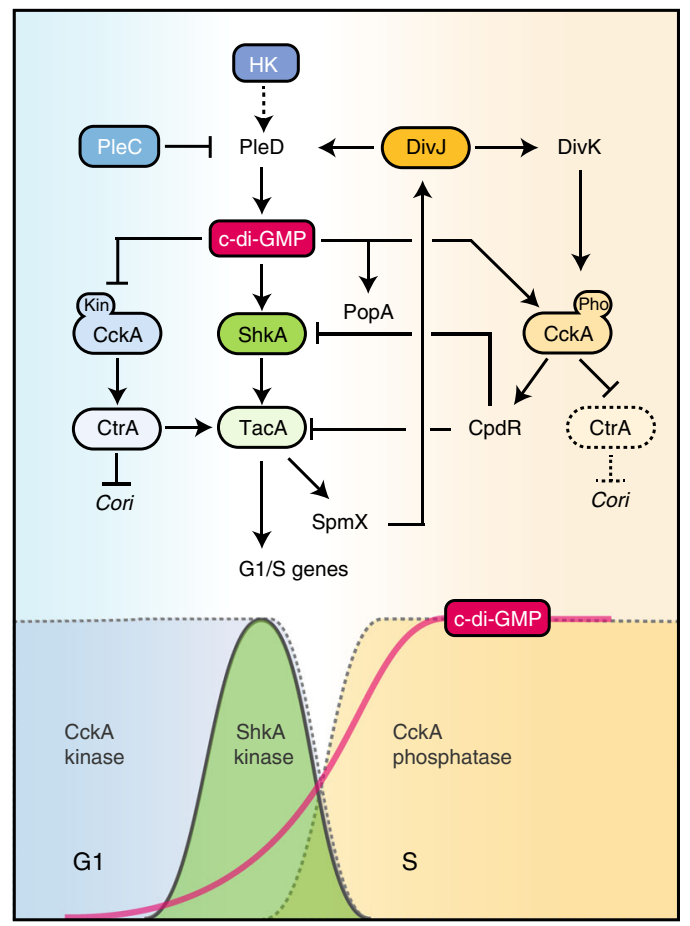

b
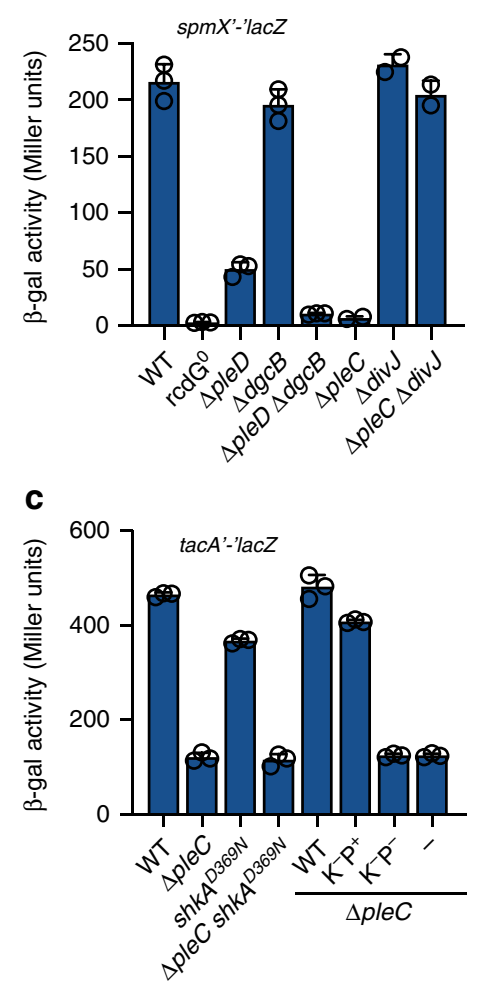

d

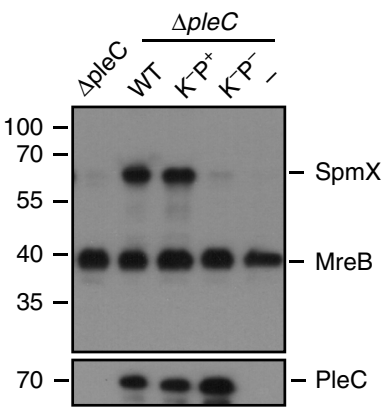

$\mathbf{e}$
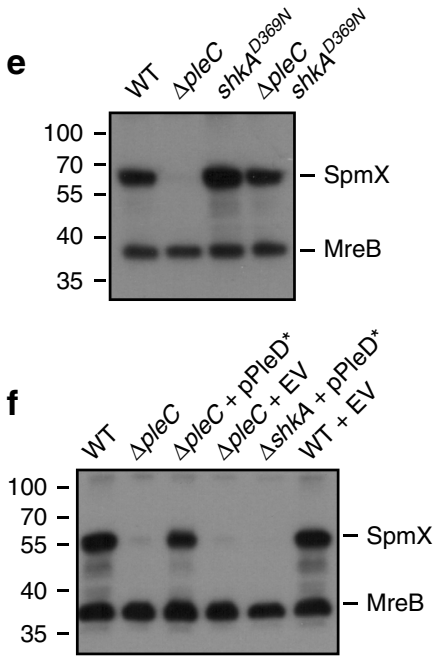

Fig. 5 C-di-GMP-dependent ShkA activation requires PleC and PleD. a Model of ShkA-TacA phosphorelay activation and its contribution to G1/S transition. The upper part shows the intercalated network of kinases (PleC, DivJ, ShkA, CckA) and response regulators (PleD, TacA, DivK) that contribute to G1/S transition. HK indicates a hypothetical histidine kinase that controls the activity of PleD and possibly DivK (stippled lines) and by that acts as kickstarter for G1 exit. HK expression is postulated to be CtrA-mediated (stippled line). The initial, HK-PleD-mediated increase of c-di-GMP activates the ShkATacA pathway, resulting in a SpmX-DivJ-PleD-mediated further boost of c-di-GMP concentration, which activates the CckA phosphatase and PopA. Note that ShkA has a five- to tenfold higher affinity for c-di-GMP compared with CckA and PopA. The block of replication initiation by binding of activated CtrA to the chromosomal origin of replication (Cori) is indicated. The lower part of the graph indicates time windows of CckA kinase and phosphatase as well as TacA kinase activity. The gradual increase of c-di-GMP during G1/S is indicated. $\mathbf{b}$ Activity of the spmX promoter in strains harboring a spm $X^{\prime}-1 / a c Z$ reporter fusion (pAK502-spmX). Shown are mean values and standard deviations $(N=3)$. c Activity of the tacA promoter in strains harboring a tac $A^{\prime}$-'lac $Z$ fusion (pAK502-tacA). Strains with a chromosomal pleC deletion express wild type and mutant pleC alleles from plasmid pQF under control of their native promoter. PleC(F778L): Kinase-/phosphatase+ (K-P+); PleC(T614R) kinase-/phosphatase- (K-P-); - indicates the empty vector control (plasmid $\mathrm{pQF}$ ). Shown are mean values and standard deviations $(\mathrm{N}=3)$. $\mathbf{d}$ Immunoblots of selected strains shown in $\mathbf{c}$ probed with anti-SpmX and anti-MreB antibodies (top) or anti-PleC antibodies (bottom). e Immunoblots of selected strains shown in c probed with anti-SpmX and anti-MreB antibodies. f Immunoblots of indicated strains probed with anti-SpmX and anti-MreB antibodies. pPleD indicates a pMR20-based plasmid (pPA114-47) expressing the constitutively active, phosphorylation-independent PleD* allele. EV denotes the empty plasmid control (pMR20). Source data are provided as a Source Data file.

activity upon S-phase entry (Fig. 5a). Although the nature of this kinase is currently unknown, we speculate that its expression or activity is CtrA-dependent and that it plays a key role in orchestrating exit from G1 as it may not only serve to activate $\mathrm{PleD}$ and provide the initial boost of c-di-GMP but may also contribute to the activation of DivK, a factor required for the CckA kinase/phosphatase switch. The essential nature of DivK but not of DivJ, its only known activating kinase, argues for regulatory redundancy in the upstream components required to boost DivK phosphorylation during G1/S (Fig. 5a).

We postulate that in its default state, the ShkA kinase is inhibited by the C-terminal REC2 domain and that c-di-GMP binding liberates the kinase by interfering with this off-state conformation. A ShkA variant lacking the REC2 domain is active without c-di-GMP ${ }^{13}$. Similarly, mutations of residues important for REC2 function lead to constitutive, c-di-GMP-independent autokinase activity. Thus, the conserved DDR motif in the REC1REC2 linker likely serves to lock ShkA in the inactive state when no c-di-GMP is present. REC2 may be closely tethered to REC1 in the inactive state through an interaction of the DDR linker motif with REC1, a conformation that may prevent the productive interaction of the catalytic CA with the DHp domain. We hypothesize that binding of c-di-GMP to REC1 interferes with this tethering, thereby liberating REC2 and facilitating the productive interaction between CA and DHp for autophosphorylation and eventually for phosphotransfer between DHp and REC2. This model is strongly supported by an accompanying structural analysis of ShkA 22 .

These findings demonstrate that degenerate REC domains, also called pseudo-receiver domains, can function as docking sites for small regulatory molecules. Hybrid histidine kinases with pseudoreceiver domains located between the $\mathrm{CA}$ and the phosphorylated receiver domain are widespread in bacteria and include the wellstudied virulence factors of the GacS/BarA family ${ }^{23,24}$ or the global stress regulator $\mathrm{RcsC}^{25,26}$ (Supplementary Fig. 5). For instance, the pseudo-receiver of $\mathrm{RcsC}$ can only be recognized by 
structural comparison ${ }^{25}$, arguing that primary structure-based searches largely underestimate the actual number of these modules. It is possible that pseudo-receiver domains generally serve as binding sites for metabolites or small signaling molecule thereby modulating kinase or phosphatase activity of such key bacterial regulators. In line with this, prominent examples of kinases harboring pseudo-receiver domains like GacS in P. aeruginosa or BarA and $\mathrm{RcsC}$ in E. coli are part of complex signaling cascades that globally regulate bacterial physiology and behavior. Careful scrutiny of the exact function of these domains will be essential to improve our understanding of such important regulatory nodes in bacteria.

\section{Methods}

Growth conditions. Caulobacter crescentus was grown in PYE $(0.2 \%$ [w/v] bacto peptone, $0.1 \%\left[\mathrm{w} / \mathrm{v}\right.$ ] yeast extract, $0.8 \mathrm{mM} \mathrm{MgSO}_{4}, 0.5 \mathrm{mM} \mathrm{CaCl}_{2}$ ) or defined $\mathrm{M} 2 \mathrm{G}$ (12.2 $\mathrm{mM} \mathrm{Na}_{2} \mathrm{HPO}_{4}, 7.8 \mathrm{mM} \mathrm{KH}_{2} \mathrm{PO}_{4}, 9.3 \mathrm{mM} \mathrm{NH}_{4} \mathrm{Cl}, 0.5 \mathrm{mM} \mathrm{MgSO}_{4}, 0.5 \mathrm{mM}$ $\mathrm{CaCl}_{2}, 20 \mu \mathrm{M} \mathrm{FeSO}_{4}, 0.2 \%$ [w/v] D-glucose) medium at $30^{\circ} \mathrm{C}$. Growth in lowphosphate medium (PYETris) was performed by replacing the phosphate buffer components in $\mathrm{M} 2 \mathrm{G}$ with $20 \mathrm{mM}$ Tris- $\mathrm{HCl}$ (pH 7.0) ${ }^{12,29}$. Standard plates contained $1.5 \%[\mathrm{w} / \mathrm{v}]$ agar. Motility was assayed on PYE plates containing $0.3 \%$ agar. Escherichia coli $\mathrm{DH} 5 \mathrm{a}, \mathrm{DH} 10 \mathrm{~B}$, or TOP10 were used for cloning and were routinely cultivated in LB-Miller at $37^{\circ} \mathrm{C}$. When appropriate, media were supplemented with antibiotics at the following concentrations unless stated otherwise (liquid/solid media for C. crescentus; liquid/solid media for E. coli; in $\mu \mathrm{g} \mathrm{ml}^{-1}$ ): kanamycin (5/ $20 ; 30 / 50)$, oxytetracycline $(2.5 / 5 ; 12.5 / 12.5)$, chloramphenicol $(1 / 2 ; 20 / 30)$, gentamycin $(1 / 5 ; 20 / 20)$, spectinomycin $(25 / 50 ;-/-)$, streptomycin $(5 / 5 ;-/-)$, ampicillin $(-/-; 100 / 100)$, nalidixic acid $(15 / 20 ;-/-)$. Isopropy- $\beta$-Dthiogalactopyranoside (IPTG), 4-hydroxy-3-methoxybenzoic acid (vanillate), and xylose stocks were prepared in $\mathrm{ddH}_{2} \mathrm{O}$ at concentrations of $1 \mathrm{M}, 200 \mathrm{mM}$, and $20 \%$ $[\mathrm{w} / \mathrm{v}]$, respectively, and used at final concentrations of $300 \mu \mathrm{M}, 250 \mu \mathrm{M}$, and $0.2 \%$ in $C$. crescentus, if not otherwise stated. 4-isopropylbenzoic acid (cumate) stocks $(100 \mathrm{mM})$ were prepared in $100 \%$ ethanol and used at a final concentration of $100 \mu \mathrm{M}$. Note that for experiments in which $s h k A$ alleles were provided on plasmid $\mathrm{pQF}$ in trans no cumate was present since already leaky expression of the wild-type allele could complement the $\Delta s h k A$ null mutant phenotype.

Strains and plasmids. Plasmids are listed in Supplementary Data 2. Oligonucleotides used for plasmid construction were purchased from Sigma and are listed in Supplementary Data 3. Plasmids were constructed as follows:

pAK503: $n p t I I$ without RBS and start codon was PCR-amplified from pAK405 using primers $8959 / 9495$, the product was digested with $B s r \mathrm{GI} / \mathrm{XbaI}$ and cloned into pAK501 cut with $A c c 65 \mathrm{I} / \mathrm{XbaI}$.

pAK502-tacA: the spmX promoter and part of the codon sequence were PCRamplified from $C$. crescentus gDNA using primers 10015/10016, the product was digested with $\mathrm{XbaI} / K p n \mathrm{I}$ and cloned into pAK502 digested with the same enzymes. pAK502-spmX: the spmX promoter and part of the codon sequence were PCRamplified from C. crescentus gDNA using primers 8966/9064, the product was digested with $\mathrm{XbaI} / K p n \mathrm{I}$ and cloned into pAK502 digested with the same enzymes.

pAK503-spmX: the spmX promoter and part of the codon sequence were PCRamplified from $C$. crescentus gDNA using primers 8966/9064, the product was digested with $\mathrm{XbaI} / K p n \mathrm{I}$ and cloned into pAK503 digested with the same enzymes.

pNPTStet: tet $A$ and tetR were PCR-amplified from pQF using primers 9552/ 9553, the product was digested with SpeI/NdeI and cloned into pNPTS138 digested with AseI/XbaI.

pNPTStet-ampG: part of $a m p G$ located downstream of $s h k A$ was PCR-amplified from C. crescentus gDNA with primers 9554/9555, the product was digested with SpeI/KpnI and cloned into pNPTStet digested with the same enzymes.

pET28a-shkA: shkA was PCR-amplified from C. crescentus gDNA with primers 9745/9746, the product was digested with $N d e \mathrm{I} / E c o$ RI and cloned into pET28a digested with the same enzymes.

pET28a-shkA(D369N): shkA(D369N) was amplified from C. crescentus strain UJ9618 by colony-PCR using primers $9745 / 9746$, the product was digested with $N d e I / E c o$ RI and cloned into pET28a digested with the same enzymes.

pQF-shkA: shkA was PCR-amplified from C. crescentus gDNA with primers $9745 / 9746$, the product was digested with $N d e I / E c o R I$ and cloned into pQF digested with $A s e \mathrm{I} / E c o$ RI.

pQF-shkA(D369N): shkA(D369N) was amplified from C. crescentus strain UJ9618 by colony-PCR using primers $9745 / 9746$, the product was digested with NdeI/EcoRI and cloned into pQF digested with AseI/EcoRI.

pQF-shkA(R26A): the mutant allele was generated by SOE-PCR using pET28ashkA as template and flanking primers 669/670 and mutagenic primers 9904/9905. The product was digested with $N d e \mathrm{I} / E c o$ RI and cloned into $\mathrm{pQF}$ digested with AseI/EcoRI.

pQF-shkA(H61A): the mutant allele was generated by SOE-PCR using pET28ashkA as template and flanking primers 669/670 and mutagenic primers 9968/9969.
The product was digested with $N d e \mathrm{I} / E c o$ RI and cloned into pQF digested with AseI/EcoRI.

pQF-shkA(R74A): the mutant allele was generated by SOE-PCR using pET28ashkA as template and flanking primers 669/670 and mutagenic primers 9970/9971. The product was digested with $N d e \mathrm{I} / E c o$ RI and cloned into $\mathrm{pQF}$ digested with AseI/EcoRI. This shkA allele contains a second mutation, A451V.

pQF-shkA(W113A): the mutant allele was generated by SOE-PCR using pET28a-shkA as template and flanking primers $669 / 670$ and mutagenic primers 9906/9907. The product was digested with $N d e I / E c o R I$ and cloned into pQF digested with AseI/EcoRI. This shkA allele contains a second mutation, A451V. pQF-shkA(D125N): the mutant allele was generated by SOE-PCR using pET28a-shkA as template and flanking primers $669 / 670$ and mutagenic primers $10009 / 10010$. The product was digested with $N d e \mathrm{I} / E c o$ RI and cloned into pQF digested with AseI/EcoRI.

pQF-shkA(R128A): the mutant allele was generated by SOE-PCR using pET28a$s h k A$ as template and flanking primers 669/670 and mutagenic primers 10005/ 10006. The product was digested with $N d e I / E c o$ RI and cloned into pQF digested with AseI/EcoRI.

pQF-shkA(R130A): the mutant allele was generated by SOE-PCR using pET28ashkA as template and flanking primers 669/670 and mutagenic primers 10007/ 10008. The product was digested with $N d e I / E c o R I$ and cloned into pQF digested with AseI/EcoRI.

pQF-shkA(R128A, R130A): the mutant allele was generated by SOE-PCR using pET28a-shkA as template and flanking primers $669 / 670$ and mutagenic primers 9908/9909. The product was digested with NdeI/EcoRI and cloned into pQF digested with AseI/EcoRI.

pQF-shkA(R177A): the mutant allele was generated by SOE-PCR using pET28ashkA as template and flanking primers $669 / 670$ and mutagenic primers $9910 / 9911$ The product was digested with $N d e \mathrm{I} / E c o \mathrm{RI}$ and cloned into $\mathrm{pQF}$ digested with AseI/EcoRI. This shkA allele contains a second mutation, P233L.

pQF-shkA(F230A, F234A): the mutant allele was generated by SOE-PCR using pET28a-shkA as template and flanking primers $669 / 670$ and mutagenic primers $9972 / 9973$. The product was digested with $N d e I / E c o R I$ and cloned into pQF digested with AseI/EcoRI.

pQF-shkA(D297N): the mutant allele was generated by SOE-PCR using pET28a-shkA as template and flanking primers $669 / 670$ and mutagenic primers 9974/9975. The product was digested with $N d e \mathrm{I} / E c o R I$ and cloned into pQF digested with AseI/EcoRI.

pQF-shkA(P321A): the mutant allele was generated by SOE-PCR using pET28a$\operatorname{shkA}$ as template and flanking primers 669/670 and mutagenic primers 10013/ 10014. The product was digested with $N d e \mathrm{I} / E c o$ RI and cloned into pQF digested with AseI/EcoRI

pQF-shkA(R324A): the mutant allele was generated by SOE-PCR using pET28a$\operatorname{shkA}$ as template and flanking primers $669 / 670$ and mutagenic primers 9912/9913 The product was digested with $N d e \mathrm{I} / E c o$ RI and cloned into pQF digested with AseI/EcoRI.

pQF-shkA(D325N): the mutant allele was generated by SOE-PCR using pET28a-shkA as template and flanking primers $669 / 670$ and mutagenic primers 10011/10012. The product was digested with $N d e I / E c o R I$ and cloned into pQF digested with AseI/EcoRI

pQF-shkA(I327A): the mutant allele was generated by SOE-PCR using pET28ashkA as template and flanking primers 669/670 and mutagenic primers 9976/9977. The product was digested with $N d e \mathrm{I} / E c o$ RI and cloned into pQF digested with AseI/EcoRI. This shkA allele contains a second mutation, A451V.

pQF-shkA(Y338A): the mutant allele was generated by SOE-PCR using pET28ashkA as template and flanking primers $669 / 670$ and mutagenic primers $9978 / 9979$ The product was digested with $N d e \mathrm{I} / E c o \mathrm{RI}$ and cloned into $\mathrm{pQF}$ digested with AseI/EcoRI.

pQF-shkA(R344A): the mutant allele was generated by SOE-PCR using pET28a$\operatorname{shkA}$ as template and flanking primers 669/670 and mutagenic primers 9914/9915 The product was digested with $N d e I / E c o R I$ and cloned into pQF digested with AseI/EcoRI. This shkA allele contains a second mutation, A451V.

pQF-shkA(E402A, G403A): the mutant allele was generated by SOE-PCR using pET28a-shkA as template and flanking primers $669 / 670$ and mutagenic primers $9980 / 9981$. The product was digested with $N d e I / E c o R I$ and cloned into pQF digested with $A s e \mathrm{I} / E c o$ RI.

pQF-shkA(C404A): the mutant allele was generated by SOE-PCR using pET28ashkA as template and flanking primers 669/670 and mutagenic primers 9986/9987. The product was digested with $N d e \mathrm{I} / E c o$ RI and cloned into pQF digested with AseI/EcoRI.

pQF-shkA(D430N): the mutant allele was generated by SOE-PCR using pET28a-shkA as template and flanking primers $669 / 670$ and mutagenic primers $10028 / 10029$. The product was digested with $N d e \mathrm{I} / E c o R I$ and cloned into pQF digested with $A s e \mathrm{I} / E c o$ RI

pQF-shkA(D465A): the mutant allele was generated by SOE-PCR using pET28a-shkA as template and flanking primers 669/670 and mutagenic primers 9982/9983. The product was digested with NdeI/EcoRI and cloned into pQF digested with $A s e \mathrm{I} / E c o$ RI. This shkA allele contains a second mutation, A451V.

pQF-shkA(C469A): the mutant allele was generated by SOE-PCR using pET28ashkA as template and flanking primers 669/670 and mutagenic primers 9984/9985. 
The product was digested with $N d e \mathrm{I} / E c o \mathrm{RI}$ and cloned into $\mathrm{pQF}$ digested with AseI/EcoRI. This shkA allele contains a second mutation, $A 451 \mathrm{~V}$.

pQF-shkA(K341): the mutant allele was generated by SOE-PCR using pET28ashkA as template and flanking primers 669/670 and mutagenic primers 11171/ 11172. The product was digested with NdeI/EcoRI and cloned into pQF digested with AseI/EcoRI

pQF-shkA(P342A): the mutant allele was generated by SOE-PCR using pET28ashkA as template and flanking primers 669/670 and mutagenic primers 11173/ 11174. The product was digested with $N d e I / E c o R I$ and cloned into $\mathrm{pQF}$ digested with AseI/EcoRI

pQF-shkA(L343A): the mutant allele was generated by SOE-PCR using pET28ashkA as template and flanking primers 669/670 and mutagenic primers $11175 /$ 11176. The product was digested with NdeI/EcoRI and cloned into pQF digested with AseI/EcoRI

pQF-shkA(I340A): the mutant allele was generated by SOE-PCR using pET28ashkA as template and flanking primers 669/670 and mutagenic primers 11177/ 11178. The product was digested with NdeI/EcoRI and cloned into pQF digested with AseI/EcoRI

pQF-shkA(L338A): the mutant allele was generated by SOE-PCR using pET28ashkA as template and flanking primers 669/670 and mutagenic primers 11179/ 11180 . The product was digested with $\mathrm{NdeI} / E c o \mathrm{RI}$ and cloned into $\mathrm{pQF}$ digested with AseI/EcoRI.

pQF-shkA(G337A): the mutant allele was generated by SOE-PCR using pET28a-shkA as template and flanking primers $669 / 670$ and mutagenic primers $11181 / 11182$. The product was digested with NdeI/EcoRI and cloned into pQF digested with $A s e \mathrm{I} / E c o$ RI

pQF-shkA(K331A): the mutant allele was generated by SOE-PCR using pET28a-shkA as template and flanking primers $669 / 670$ and mutagenic primers $11183 / 11184$. The product was digested with NdeI/EcoRI and cloned into pQF digested with $A s e \mathrm{I} / E c o$ RI.

pQF-shkA(S347A): the mutant allele was generated by SOE-PCR using pET28ashkA as template and flanking primers $669 / 670$ and mutagenic primers $11185 /$ 11186. The product was digested with NdeI/EcoRI and cloned into pQF digested with AseI/EcoRI.

pQF-shkA(Q351A): the mutant allele was generated by SOE-PCR using pET28a-shkA as template and flanking primers $669 / 670$ and mutagenic primers $11187 / 11188$. The product was digested with $N d e \mathrm{I} / E c o$ RI and cloned into pQF digested with $A s e \mathrm{I} / E c o$ RI.

pQF-shkA(D369N, H61A): a fragment harboring part of shkA was released from pQF-shkA(H61A) by digestion with SpeI/PstI and cloned into pQF-shkA(D369N) digested with the same enzymes.

pQF-shkA(D369N, R128A): a fragment harboring part of shkA was released from pQF-shkA(R128A) by digestion with SpeI/PstI and cloned into pQF-shkA $(D 369 N)$ digested with the same enzymes.

pQF-shkA(D369N, R130): a fragment harboring part of shkA was released from pQF-shkA(R130A) by digestion with SpeI/PstI and cloned into pQF-shkA(D369N) digested with the same enzymes.

pQF-shkA(D369N, R128A, R130A): a fragment harboring part of shkA was released from pQF-shkA(R128A, R130A) by digestion with SpeI/PstI and cloned into $\mathrm{pQF}-\operatorname{sh} k A(D 369 N)$ digested with the same enzymes.

pQF-shkA(D369N, F230A, F234A): a fragment harboring part of shkA was released from pQF-shkA(F230A, F234A) by digestion with SpeI/PstI and cloned into $\mathrm{pQF}-\operatorname{sh} k A(D 369 N)$ digested with the same enzymes.

pQF-shkA(D369N, R128A): a fragment harboring part of shkA was released from pQF-shkA(R128A) by digestion with SpeI/PstI and cloned into pQF-shkA $(D 369 N)$ digested with the same enzymes.

pQF-shkA(D369N, R324A): a fragment harboring part of shkA was released from $\mathrm{pQF}-\mathrm{sh} k A(R 324 A)$ by digestion with SpeI/PstI and cloned into pQF-shkA $(D 369 N)$ digested with the same enzymes.

pQF-shkA(D369N, Y338A): a fragment harboring part of shkA was released from pQF-shkA(Y338A) by digestion with SpeI/PstI and cloned into pQF-shkA $(D 369 N)$ digested with the same enzymes.

pET28a-shkA(D430N): the mutant allele was generated by SOE-PCR using pET28a-shkA as template and flanking primers $669 / 670$ and mutagenic primers 10028/10029. The product was digested with NdeI/EcoRI and cloned into pET28a digested with $\mathrm{NdeI} / E c o$ RI.

pET28a-shkA(E386Q, D387N): the mutant allele was generated by SOE-PCR using pET28a-shkA as template and flanking primers 669/670 and mutagenic primers 10026/10027. The product was digested with $N d e \mathrm{I} / E c o$ RI and cloned into pET28a digested with $\mathrm{NdeI} / E c o$ RI.

pET28a-shkA(K480R): the mutant allele was generated by SOE-PCR using pET28a-shkA as template and flanking primers $669 / 670$ and mutagenic primers 10024/10025. The product was digested with NdeI/EcoRI and cloned into pET28a digested with $N d e I / E c o$ RI.

pET28a-shkA(R324A): a fragment harboring part of shkA was released from pQF-shkA(R324A) by digestion with AscI/PstI and cloned into pET28a-shkA digested with the same enzymes.

pET28a-shkA(D369N, R324A): a fragment harboring part of shkA was released from pQF-shkA(R324A) by digestion with AscI/PstI and cloned into pET28a-shkA $(D 369 N)$ digested with the same enzymes.
pET28a-shkA(Y338A): a fragment harboring part of shkA was released from pQF-shkA(Y338A) by digestion with AscI/PstI and cloned into pET28a-shkA digested with the same enzymes.

pET28a-shkA(D369N, Y338A): a fragment harboring part of $s h k A$ was released from $\mathrm{pQF}-s h k A(Y 338 A)$ by digestion with $A s c \mathrm{I} / \mathrm{PstI}$ and cloned into $\mathrm{pET} 28 \mathrm{a}-\mathrm{sh} k A$ $(D 369 N)$ digested with the same enzymes.

pET28a-shkA(R128A, R130A): the mutant allele was generated by SOE-PCR using pET28a-shkA as template and flanking primers $669 / 670$ and mutagenic primers 9908/9909. The product was digested with NdeI/EcoRI and cloned into pET28a digested with the same enzymes.

pET28a-shkA-REC1: a fragment of shkA encoding REC1 was PCR-amplified from C. crescentus $\mathrm{gDNA}$ with primers $10862 / 10866$, the product was digested with $\mathrm{NdeI} / K p n \mathrm{I}$ and cloned into pUC18 digested with the same enzymes. A NdeI/EcoRI from the resulting plasmid was subcloned into pET28a digested with the same enzymes.

pQF-pleC: a DNA fragment including pleC ORF and the mapped pleC promoter as PCR-amplified from plasmid pJS14-pleC with primers 10781/10783, the product was digested with $\mathrm{SpeI} / \mathrm{Kp} n \mathrm{I}$ and cloned into $\mathrm{pQF}$ digested with the same enzymes. pQF-pleC $(K-P-)$ : a DNA fragment including pleC(T614R) ORF and the mapped pleC promoter as PCR-amplified from plasmid pJS14-pleC(T615R) with primers 10781/10783, the product was digested with SpeI/KpnI and cloned into pQF digested with the same enzymes.

pQF-pleC $(K-P+)$ : a DNA fragment including pleC(F778L) ORF and the mapped pleC promoter as PCR-amplified from plasmid pJS14-pleC(F778L) with primers 10781/10783, the product was digested with SpeI/KpnI and cloned into $\mathrm{pQF}$ digested with the same enzymes.

pdivJ-mCherry: the 3' part of divJ was PCR-amplified from C. crescentus gDNA with primers 10205/10206, the product was digested with KpnI/AgeI and cloned into pCHYC-4 digested with the same enzymes.

pNPTS138- $\triangle$ tacA: two DNA fragments flanking the tacA ORF were PCRamplified from C. crescentus gDNA. Fragment 1 was amplified with primers 6535/ 6098, then digested with PstI/KpnI. Fragment 2 was amplified with primers 6099/ 6536, then digested with KpnI/EcoRI. The two fragments were ligated to pNPTS138 digested with $P s t \mathrm{I} / E c o$ RI

pNPTS138- $\Delta$ shkA: two DNA fragments flanking the shkA ORF were PCRamplified from C. crescentus gDNA. Fragment 1 was amplified with primers 6085/ 6086, then digested with $P s t \mathrm{I} / \mathrm{KpnI}$. Fragment 2 was amplified with primers 6087/ 6088, then digested with $K p n I / E c o$ RI. The two fragments were ligated to pNPTS138 digested with $P s t \mathrm{I} / E c o$ RI

pNPTS138-3xFLAG-tacA: the upstream region of the tacA ORF was PCRamplified from C. crescentus gDNA with primers 6438/6439 (fragment 1). A $5^{\prime}$ part of tacA was PCR-amplified from C. crescentus gDNA with primers 6440/7604 (fragment 2), and then used as a template with primers 6441/7604 to insert the coding sequence of the 3xFlag-Tag (fragment 3). SOE-PCR was performed with fragment 1 and 3 as template and primers 6438/7604. The resulting product was then digested with PstI/EcoRI and ligated into pNPTS138 digested with the same enzymes.

PNPTS138-3xFLAG-shkA: the upstream region of the shkA ORF was PCRamplified from C. crescentus gDNA with primers 6442/6443 (fragment 1). A 5' part of shkA was PCR-amplified from C. crescentus gDNA with primers 6444/7605 (fragment 2), and then used as a template with primers 6445/7605 to insert the coding sequence of the 3xFlag-Tag (fragment 3). SOE-PCR was performed with fragment 1 and 3 as template and primers 6442/7605. The resulting product was then digested with $P s t \mathrm{I} / E c o R I$ and ligated into pNPTS138 digested with the same enzymes.

pNPTS138-shkA(DD): the $5^{\prime}$ region of $s h k A$ was PCR-amplified from $C$. crescentus $\mathrm{gDNA}$ with primers $8169 / 8170$ (fragment 1). The downstream region of the shkA ORF was PCR-amplified from C. crescentus gDNA with primers 8171/ 8172 (fragment 2). SOE-PCR was performed with the two fragments as template and primers 8169/8172. The resulting product was then digested with BamHI/ $E c o$ RI and ligated into pNPTS138 digested with the same enzymes.

pNPTS138-tac $A(D D)$ : the $5^{\prime}$ region of $t a c A$ was PCR-amplified from $C$. crescentus gDNA with primers 7925/7716 (fragment 1). The downstream region of the tacA ORF was PCR-amplified from C. crescentus gDNA with primers 7717/ 7718 (fragment 2). SOE-PCR was performed with the two fragments as template and primers 7925/7718. The resulting product was then digested with HindIII/ EcoRI and ligated into pNPTS138 digested with the same enzymes.

pNPTS138-tacA(D54E): the mutant allele was generated by SOE-PCR using $C$. crescentus gDNA as template and flanking primers 7958/7961 and mutagenic primers 7959/7960. The product was digested with PstI/EcoRI and cloned into pNPTS138 digested with the same enzymes.

pNPTS138- $\Delta$ spmX: two DNA fragments flanking the spmX ORF were PCRamplified from C. crescentus gDNA. Fragment 1 was amplified with primers 7268 / 7269 , fragment 2 was amplified with primers 7270/7271. The two fragments were joined by SOE-PCR using primers 7268/7271, digested with PstI/EcoRI, and ligated into pNPTS138 digested with the same enzymes.

pNPTS138- $\triangle C C 0168$ : the region upstream of CC0168 was amplified from $C$. crescentus NA1000 gDNA with primer pairs 3490 and 3491. The PCR product was cloned into pGEM-T Easy, sequenced, and cut out with HindIII and KpnI. The region downstream of CC0168 was amplified from C. crescentus NA1000 gDNA 
with primer pairs 3492 and 3493. The PCR product was cloned into pGEM-T Easy, sequenced and cut out with BamHI and KpnI. Both cut PCR products were ligated in a triple ligation with pNPTS138 digested with HindIII and BamHI.

pET28a-His-shkA: shkA was PCR-amplified from C. crescentus gDNA with primers 5988/5486, the product was digested with NdeI/HindIII and cloned into pET28a digested with the same enzymes.

pET28a-His-shpA: $\operatorname{sh} p A$ was PCR-amplified from C. crescentus gDNA with primers 6015/6016, the product was digested with HindIII/EcoRI and cloned into pET28a digested with the same enzymes.

pET28a-His-tacA-RD: the sequence of tacA encoding the receiver domain was PCR-amplified from C. crescentus gDNA with primers 7925/6013, the product was digested with HindIII/EcoRI and cloned into pET28a digested with the same enzymes.

pET32b-Trx-His-shkA-HK: the sequence of shkA encoding the kinase catalytic core including the REC1 domain was PCR-amplified from C. crescentus gDNA with primers 6110/6011, the product was digested with HindIII/EcoRI and cloned into pET32b digested with the same enzymes.

pET32b-Trx-His-shkA-RD2: the sequence of shkA encoding the REC2 domain was PCR-amplified from C. crescentus gDNA with primers 6109/6009, the product was digested with HindIII/EcoRI and cloned into pET32b digested with the same enzymes.

pMT687-tacA: tacA was PCR-amplified from C. crescentus gDNA with primers $5487 / 5490$, the product was digested with $N d e I / K p n I$ and cloned into pMT687 digested with the same enzymes.

pMT687-tacA(D54E): the mutant allele was generated by SOE-PCR using $C$. crescentus gDNA as template and flanking primers 5487/5490 and mutagenic primers 5488/5489. The product was digested with NdeI/KpnI and cloned into pNPTS138 digested with the same enzymes.

pQF-PA5295: PA5295 was PCR-amplified from pRV-PA5295 with primers 8974/8975, the product was digested with HindIII/KpnI and cloned into pMT687 digested with the same enzymes.

pQF-PA5295(AAL): PA5295(AAL) was PCR-amplified from pRV-PA5295

(AAL) with primers 8974/8975, the product was digested with HindIII/KpnI and cloned into pMT687 digested with the same enzymes.

pRKlac290-spmX: the promoter region of spmX was PCR-amplified from C. crescentus gDNA with primers 6864/6865. The resulting product was digested with EcoRI/PstI and ligated into pRKlac290 digested with the same enzymes.

pRKlac290-staR: the promoter region of staR was PCR-amplified from C. crescentus gDNA with primers 6621/6622. The resulting product was digested with EcoRI/PstI and ligated into pRKlac290 digested with the same enzymes.

pAH10: pUC19 was digested with HindIII and PciI to integrate the following annealed oligos 5786/5903/5904/5905 downstream of the MCS.

pAH87: pMT375 was digested with $\mathrm{XbaI}$ and its insert was replaced with the NsiI-PacI cassette of pAH10 amplified with 7284 and 7287 digested with NheI/SpeI. pAH99: dendra2 was PCR-amplified from pDHL851 using primers 7503 and 7507, which also introduced a stop codon and the RBS of pQE70 upstream of dendra2. The product was digested with HindIII and PacI and ligated into pAH87 digested with the same restriction enzymes.

pAH111: the promoter region of spmX was PCR-amplified from C. crescentus gDNA with primers 6864 and 7512, digested with EcoRI/HindIII and ligated into pAH99 digested with the same enzymes.

pAH139: dendra2-ssrA was generated by PCR from pDHL851 with primers 7503 and 6478. The resulting product was digested with HindIII/XhoI and ligated into pAH111 digested with the same enzymes.

Plasmid was delivered into C. crescentus by electroporation or triparental mating using LS980 as helper strain. $\varphi$ CR30 was used for generalized transduction ${ }^{27}$. Strains are listed in Supplementary Data 4. In general, C. crescentus mutant strains were constructed by a two-step homologous recombination selection/counterselection procedure using pNPTS138. Alternatively, pNPTS138based plasmids used for construction of a specific mutant strain were first integrated in this strain and then the mutation was moved into a clean background using $\varphi$ CR30-mediated generalized transduction, followed by resolution of the merodiploid by counterselection on PYE plates containing $0.3 \%[\mathrm{w} / \mathrm{v}]$ sucrose

Synchronization. Strains were grown overnight in $5 \mathrm{ml}$ PYE supplemented with appropriate antibiotics and inducers in a roller drum at $30^{\circ} \mathrm{C}$. The next day, cultures were diluted 20 -fold in $25 \mathrm{ml} \mathrm{M} 2 \mathrm{G}$ and allowed to grow on a rocking shaker at $30^{\circ} \mathrm{C}$ until they reached an $\mathrm{OD}_{660}$ of $0.4-0.6$. These cultures were then subcultured in $300 \mathrm{ml}$ M2G such that the cultures reached an $\mathrm{OD}_{660}$ of 0.6 after overnight incubation on a rocking shaker $(160 \mathrm{rpm})$ at $30^{\circ} \mathrm{C}$. Cells were harvested $\left(5000 \times g, 10 \mathrm{~min}, 4^{\circ} \mathrm{C}\right)$, the supernatant was aspirated, cells were resuspended in $40 \mathrm{ml}$ cold phosphate buffer $\left(12.3 \mathrm{mM} \mathrm{Na}_{2} \mathrm{HPO}_{4}, 7.8 \mathrm{mM} \mathrm{K \textrm {K } _ { 2 }} \mathrm{PO}_{4}\right)$ or in cold $\mathrm{M} 2 \mathrm{G}$ for experiments shown in Fig. $3 \mathrm{C}$ and kept at $4{ }^{\circ} \mathrm{C}$ for the rest of the procedure. $20 \mathrm{ml}$ cold Ludox were added and the suspension mixed thoroughly before being transferred to precooled glass Corex tubes and spun to separate the swarmer from the stalked cells $\left(9780 \times g, 35 \mathrm{~min}, 4^{\circ} \mathrm{C}\right)$. The top layer of cells was aspirated, the swarmer band moved to a clean precooled Corex tube and washed twice with $30 \mathrm{ml}$ of either cold phosphate buffer or M2G (see above) by centrifugation $\left(8000 \times g, 10 \mathrm{~min}, 4^{\circ} \mathrm{C}\right)$. Cells were released to a flask with pre-warmed M2G at an $\mathrm{OD}_{660}$ of $\sim 0.3$ and incubated in a water bath $\left(30^{\circ} \mathrm{C}, 150 \mathrm{rpm}\right)$. Samples were taken at indicated time points in precooled tubes and harvested at max. speed using a cooled table-top centrifuge. The pellets were immediately snap-frozen in liquid nitrogen and stored at $-20^{\circ} \mathrm{C}$ until further processing.

Phos-tag PAGE. Samples from mixed cultures grown in PYE were taken in exponential phase, and sampling of synchronized cultures is described above. An equivalent of $0.5 \mathrm{ml}$ cell culture at an $\mathrm{OD}_{660}$ of 0.5 was harvested by centrifugation in a table-top centrifuge at max speed and the pellets were snap-frozen in liquid nitrogen and stored at $-20^{\circ} \mathrm{C}$. Cells were lysed in $100 \mu$ lysis buffer $(5 \mathrm{ml}$ contain: $10 \mathrm{mM}$ Tris- $\mathrm{HCl} \mathrm{pH} \mathrm{7.5,} \mathrm{4 \%} \mathrm{SDS,} 1$ PhosSTOP tablet [Roche], a scoop of DNase I [NEB]) for $5 \mathrm{~min}$ at room temperature. After spinning the samples for $5 \mathrm{~min}$ at max speed at room temperature in a table-top centrifuge, $80 \mu \mathrm{l}$ of the cell lysate was taken up in $120 \mu \mathrm{l} 1.6 \mathrm{X}$ SDS sample buffer $(0.1 \mathrm{M}$ Tris $\mathrm{pH} 6.8,5 \%[\mathrm{v} / \mathrm{v}]$ glycerol $0.2 \%[\mathrm{w} / \mathrm{v}]$ SDS, $1 \%[\mathrm{v} / \mathrm{v}] \beta$-mercaptoethanol, $0.025 \%[\mathrm{w} / \mathrm{v}]$ bromophenol blue) and kept on ice. Subsequently, $20 \mu \mathrm{l}$ of sample were run on 12\% SDS-PAGE gels supplemented with $100 \mu \mathrm{M} \mathrm{MnCl}_{2}$ and $50 \mu \mathrm{M}$ Phos-tag acrylamide compound (Wako) for $4-5 \mathrm{~h}$ at $100 \mathrm{~V}$ at $4{ }^{\circ} \mathrm{C}$. Subsequent immunoblotting was performed as described below.

Immunoblotting. Samples from mixed cultures grown in PYE were taken in exponential phase $\left(\mathrm{OD}_{660}\right.$ of $\left.0.3-0.4\right)$ and cells were harvested by centrifugation (max speed, $1 \mathrm{~min}$, room temperature) in a table-top centrifuge. Sampling of synchronized cultures is described above. Pellets were resuspended in 1X SDS sample buffer $(62.5 \mathrm{mM}$ Tri-HCl pH 6.8, $10 \%[\mathrm{v} / \mathrm{v}]$ glycerol, $2 \%$ [w/v] SDS, $5 \%$ $[\mathrm{v} / \mathrm{v}] \beta$-mercaptoethanol, $0.001 \%[\mathrm{w} / \mathrm{v}]$ bromophenol blue) and boiled for 5-10 min before being run on 10-12.5\% SDS-PAGE or precast Mini-Protean TGX (Biorad) gels. Proteins were transferred from SDS-PAGE gels to PVDF-membranes (Immobilon-P, Millipore $0.45 \mu \mathrm{m}$ ) in transfer buffer (for 1 1: $3.03 \mathrm{~g}$ Tris base, $14.4 \mathrm{~g}$ glycine, $20 \%[\mathrm{v} / \mathrm{v}]$ ethanol, $\left.\mathrm{ddH}_{2} \mathrm{O}\right)$ using a Biorad semi-dry system $(24 \mathrm{~V}, 300 \mathrm{~mA}$, $30 \mathrm{~min}$ ) or a Biorad wet blot system $\left(100 \mathrm{~V}, 1 \mathrm{~h}, 4^{\circ} \mathrm{C}\right)$ (Fig. 3c). For immunoblotting with Phos-tag gels, gels were kept at $4^{\circ} \mathrm{C}$ and washed successively for $10 \mathrm{~min}$ with transfer buffer containing $10 \mathrm{mM}$ EDTA and transfer buffer containing $0.1 \%(\mathrm{w} / \mathrm{v})$ SDS, and transfer to PVDF-membranes (Immobilon-P, Millipore, $0.45 \mu \mathrm{m})$ was done using a Biorad wet blot system $\left(80 \mathrm{~V}, 120 \mathrm{~min}, 4^{\circ} \mathrm{C}\right)$. After transfer, membranes were blocked by incubation with blocking buffer for $1 \mathrm{~h}$ at room temperature. Blocking buffer was $1 \mathrm{X}$ PBS $(137 \mathrm{mM} \mathrm{NaCl}, 27 \mathrm{mM} \mathrm{KCl}$, $\left.81 \mathrm{mM} \mathrm{Na}_{2} \mathrm{HPO}_{4}, 18 \mathrm{mM} \mathrm{KH}_{2} \mathrm{PO}_{4}\right)$ containing $0.1 \%(\mathrm{v} / \mathrm{v})$ Tween20 and $5 \%(\mathrm{w} / \mathrm{v})$ skimmed milk powder (for experiments shown in Fig. 3c) or TBST $(20 \mathrm{mM} 1 \mathrm{M}$ Tris- $\mathrm{HCl} \mathrm{pH}$ 7.5, $150 \mathrm{mM} 5 \mathrm{M} \mathrm{NaCl}, 0.1 \%$ [v/v] Tween20) containing 5\% skimmed milk powder (all other experiments). After blocking, membranes were incubated with the primary antibody in blocking buffer for $1 \mathrm{~h}$ at room temperature or overnight at $4^{\circ} \mathrm{C}$, followed by three washes in blocking buffer $(5 \mathrm{~min}$, room temperature) and incubation with the secondary antibody in blocking buffer for $1 \mathrm{~h}$ at room temperature. Membranes were washed three to four times with blocking buffer and three times in 1X PBS (for experiments shown in Fig. 3c) before addition of ECL detection reagent (KPL LumiGLO or LumiGLO Reserve, SeraCare Life Sciences). Chemiluminescence was detected using a Fujifilm LAS-4000 Imager (Fuji) with automatic exposure time determination or by exposure of membranes to X-ray films (Fujifilm). Primary antibodies were used at the following dilutions: $\alpha-\operatorname{MreB}(1: 20,000)$ (a gift from Régis Hallez), $\alpha$-TacA $(1: 15,000)^{12}, \alpha$-SpmX $(1: 10,000)^{12}, \alpha-P l e C(1: 5000)^{28}, \alpha-\operatorname{CtrA}(1: 5000)^{29}$, M2 $\alpha$-FLAG $(1: 10,000)$ (Sigma). Secondary antibodies HRP-conjugated rabbit anti-mouse and swine anti-rabbit (Dako Cytomation, DK) were used at a 1:10,000 dilution.

Microscopy and image analysis. Cultures were grown in PYE and imaged in exponential phase $\left(\mathrm{OD}_{660}\right.$ of $\left.0.3-0.4\right)$ on $1 \%$ agarose PYE pads. Microscopy images were acquired using softWoRx 6.0 (GE Healthcare) on a DeltaVision system (GE Healthcare), equipped with a pco.edge sCMOS camera, and an UPlan FL N 100×/ 1.30 oil objective (Olympus). DivJ-mCherry localization was analyzed using Fiji software package ${ }^{30}$ with the MicrobeJ plugin ${ }^{31}$. Oufti ${ }^{32}$ was used to quantify cell length and data were analyzed in Prism 7 (GraphPad) with statistical testing using ordinary one-way ANOVA and Tukey's multiple comparison test. SpmX-mCherry localization and stalk formation (Supplementary Fig. 1) was analysed manually.

Time-lapse microscopy and image analysis. For Fig. 4 and Supplementary Fig. 7 spmX promoter activity during the cell cycle of single cells of C. crescentus wild type and selected mutants was analyzed using $\mathrm{P}_{s p m X}$-Dendra2 and time-lapse microscopy. Strains were grown overnight in $5 \mathrm{ml}$ PYE supplemented with appropriate antibiotics in a roller drum at $30^{\circ} \mathrm{C}$. On the day of the experiment, these cultures were diluted 50 -fold in $5 \mathrm{ml}$ PYE supplemented with $2.5 \mu \mathrm{g} \mathrm{m} l^{-1}$ oxytetracycline and if appropriate with $0.1 \mathrm{mM} \mathrm{IPTG}$ and allowed to grow in a roller drum at $30^{\circ} \mathrm{C}$ until they reached an $\mathrm{OD}_{660}$ of 0.2 . Cells were mounted on $1 \%$ PYE medium agar pads containing appropriate supplements sealed with a double layer of gene frames (Thermo Fisher Scientific; $1.7 \times 2.8 \mathrm{~cm}$ ) and subjected to time-lapse microscopy using softWoRx 6.0 (GE Healthcare) on a DeltaVision system (GE Healthcare), equipped with a pco.edge sCMOS camera, and an UPlan FL N 100×/1.30 oil objective (Olympus) using $0.3 \mathrm{~s}$ exposure time for phase-contrast, FITC and TRITC channels and a frame rate of 1 frame/ 15 min for different amounts of time.

For Fig. 4b, c, Supplementary Fig. 7, green Dendra2 was irreversibly photoconverted to red in the latest detectable predivisional stage $15 \mathrm{~min}$ before the 
first visible cell separation and birth of the swarmer cell using $2 \mathrm{~s}$ UV light (DAPI channel) leading to a conversion efficiency from green to red between $62-75 \%$. Image processing and analysis was done using the Fiji software package ${ }^{30}$. From every predivisional cell and its offspring (swarmer and stalked cell) the total cell fluorescence and cell area was manually measured for every time point over the course of one cell cycle and if appropriate for up to three consecutive cell cycles and the integrated density (mean intensity normalised to cell area) determined. In case of time point 0 (predivisional cell after photoconversion), the mean intensity of the predivisional was normalised to the area of the swarmer or stalked cell. Integrated density values were then normalised for background fluorescence to obtain normalised fluorescence values over the cell cycle, plotted as arbitrary units (A.U.) in Supplementary Fig. 7.

In order to determine the fraction of cells with induced spmX promoter activity in Fig. $5 c$, for individual cells the mean of all normalised fluorescence values over one cell cycle was calculated. If the mean normalised fluorescence was above $150 \mathrm{~A}$. U., cells were scored as showing induced PspmX activity; if below 150 A.U. then cells were scored as non-induced.

In Fig. 4c normalised fluorescence values over the cell cycle of each cell type were set relative to the value of the late predivisional cell after photoconversion $\left(t_{0}=1\right)$. Green Dendra2 was irreversibly photoconverted to red only once in the latest detectable predivisional stage $15 \mathrm{~min}$ before the first visible cell separation and birth of the swarmer cell of generation 1. Image analysis was done as described above. Normalised fluorescence values of each cell type over each of the three cell cycles were set relative to the first late predivisional cell after photoconversion. The slope of relative fluorescence was determined over the course of one cell cycle and cells were scored as showing induced activity if the slope $\geq 0.19$ or as not showing activity if the slope $\leq 0.19$. The fraction of cells with induced spmX promoter activity was then calculated and plotted.

The ON kinetics of $\mathrm{P}_{s p m x}$ activity in Fig. $4 \mathrm{~b}$ was determined as following: Dendra2 was photoconverted once using $2 \mathrm{~s}$ UV light (DAPI channel) in the latest detectable predivisional stage $15 \mathrm{~min}$ before the first visible cell separation and birth of the swarmer cell. Cells were normalized and evaluated as above. The time of the first visible increase of cell fluorescence in the swarmer cell (green line) and the stalked cell (gray line) cells with slope $\geq 0.075$ was plotted as fraction of cells with induced spmX promoter activity over time. For inferred OFF kinetics (blue dashed line), cells at different cell cycle stages were photoconverted once using $2 \mathrm{~s}$ UV light (DAPI channel), normalized as above and the increase of fluorescence within 15-min intervals was determined using the first time point after visible cell separation as reference. Fraction of cells scored as having induced $\operatorname{spmX}$ promoter activity over the cell cycle was plotted over time. Results were plotted using Prism 7 (GraphPad)

$\boldsymbol{\beta}$-Galactosidase assays. For $\beta$-galactosidase assays strains harboring pAK502based lacZ reporter plasmids were grown in $2 \mathrm{ml}$ PYE supplemented with chloramphenicol and additional antibiotics as appropriate overnight at $30^{\circ} \mathrm{C}$ in a drum roller and diluted the next day 20 -fold in $2 \mathrm{ml}$ of the same medium, followed by further incubation for an additional $4.5 \mathrm{~h}$ under the same conditions before sampling. $\beta$-Galactosidase assays were performed according to Miller ${ }^{33}$. For $\beta$ galactosidase assays strains harboring pRKlac290-based plasmids were grown overnight in $5 \mathrm{ml}$ PYE supplemented with the appropriate antibiotics in a roller drum at $30^{\circ} \mathrm{C}$. On the day of the experiment, these cultures were diluted 20 -fold in $5 \mathrm{ml}$ PYE supplemented with appropriate antibiotics and allowed to grow in a roller drum at $30^{\circ} \mathrm{C}$ until they reached an $\mathrm{OD}_{660}$ of $0.3-0.54 .1 .8 \mathrm{ml}$ culture was spun and the pellet resuspended in $1.8 \mathrm{ml} \mathrm{Z-buffer}\left(0.06 \mathrm{M} \mathrm{Na}_{2} \mathrm{HPO}_{4}, 0.04 \mathrm{M}\right.$ $\mathrm{NaH}_{2} \mathrm{PO}_{4}, 0.01 \mathrm{M} \mathrm{KCl}, 0.001 \mathrm{M} \mathrm{MgSO}_{4}, 0.05 \mathrm{M} \beta$-mercaptoethanol) of which $0.8 \mathrm{ml}$ were used for $\mathrm{OD}_{660}$ measurements and $1 \mathrm{ml}$ transferred to a chloroformstable Eppendorf tube. Subsequently, $100 \mu \mathrm{l} \mathrm{0.1 \%} \mathrm{SDS}$ and $20 \mu \mathrm{l}$ chloroform were added. Samples were vortexed for $10 \mathrm{~s}$ and incubated at room temperature for $30-60 \mathrm{~min}$. From the top aqueous layer of the mixture three times $200 \mu \mathrm{l}$ (three technical replicates) were transferred to a $96-$ well plate, $25 \mu \mathrm{l}$ ONPG $\left(4 \mathrm{mg} \mathrm{ml}^{-1}\right.$ in Z-buffer) were added to each well and consumption of ONPG was followed at $405 \mathrm{~nm}$ using a BioTek Instruments EL800 plate reader (20 reads at the fastest interval). $\beta$-galactosidase activity was calculated as the initial slope of the increase of $\mathrm{OD}_{405}$ over time at a linear range and corrected for the $\mathrm{OD}_{660}$ and volume. Activities were normalized to the activity of a wild-type strain that was included in all assays.

Flow cytometry. Cultures were inoculated from a fresh colony and strains were grown in $2 \mathrm{ml}$ PYE at $30^{\circ} \mathrm{C}$ in a drum roller $20 \mathrm{~h}$ (stationary phase) or diluted back 20 -fold in $2 \mathrm{ml}$ of the same medium after overnight incubation, followed by incubation for an additional $4.5 \mathrm{~h}$ (exponential phase). Rifampicin was added to cultures at a final concentration of $25 \mu \mathrm{g} \mathrm{ml}^{-1}$ and cultures were incubated for $2 \mathrm{~h}$ under the same conditions before cells were being fixed in cold $70 \%$ ethanol. Cells were collected by centrifugation, resuspended in $0.5 \mathrm{ml} \mathrm{FACS}$ buffer $(10 \mathrm{mM}$ Tris$\mathrm{HCl} \mathrm{pH} 7.5,1 \mathrm{mM}$ EDTA, $50 \mathrm{mM}$ sodium citrate, $0.01 \%$ [v/v] TritonX-100) containing $2.5 \mu \mathrm{l}$ RNaseA solution (Sigma) and incubated at room temperature for $30 \mathrm{~min}$, after which $50 \mu \mathrm{l}$ were transferred to $1 \mathrm{ml}$ FACS buffer containing $1.5 \mu \mathrm{l}$ YO-PRO-1 iodide (Thermo Fisher Scientific) and incubated for $2 \mathrm{~h}$ at room temperature in the dark. Data were acquired using a FACS Canto II (BD Biosciences) with $>50,000$ events recorded and analyzed with FlowJo software (FlowJo LLC). The gating strategy is outlined in Supplementary Fig. 10.
Mass spectrometry-based proteome analysis. $10^{9}$ C. crescentus cells grown in PYE to exponential phase were collected, washed twice with phosphate buffer, resuspended in $50 \mu \mathrm{l}$ lysis buffer ( $1 \%$ sodium deoxycholate, $0.1 \mathrm{M}$ ammoniumbicarbonate), reduced with $5 \mathrm{mM}$ TCEP for $15 \mathrm{~min}$ at $95^{\circ} \mathrm{C}$ and alkylated with 10 $\mathrm{mM}$ chloroacetamide for $30 \mathrm{~min}$ at $37^{\circ} \mathrm{C}$. Samples were digested with trypsin (Promega) at $37^{\circ} \mathrm{C}$ overnight (protein to trypsin ratio: 50:1) and desalted on $\mathrm{C} 18$ reversed phase spin columns according to the manufacturer's instructions (Microspin, Harvard Apparatus).

One microgram of peptides of each sample were subjected to LC-MS analysis using a dual pressure LTQ-Orbitrap Elite mass spectrometer connected to an electrospray ion source (both Thermo Fisher Scientific). Peptide separation was carried out using an EASY nLC-1000 system (Thermo Fisher Scientific) equipped with a RP-HPLC column $(75 \mu \mathrm{m} \times 30 \mathrm{~cm})$ packed in-house with $\mathrm{C} 18$ resin (ReproSil-Pur C18-AQ, $1.9 \mu \mathrm{m}$ resin; Dr Maisch GmbH, Ammerbuch-Entringen, Germany) using a linear gradient from $95 \%$ solvent A ( $0.15 \%$ formic acid, $2 \%$ acetonitrile) and $5 \%$ solvent B ( $98 \%$ acetonitrile, $0.15 \%$ formic acid) to $28 \%$ solvent $\mathrm{B}$ over $75 \mathrm{~min}$ at a flow rate of $0.2 \mu \mathrm{l} / \mathrm{min}$. The data acquisition mode was set to obtain one high resolution MS scan in the FT part of the mass spectrometer at a resolution of 240,000 full width at half-maximum (at $\mathrm{m} / z$ 400) followed by MS/MS scans in the linear ion trap of the 20 most intense ions. The charged state screening modus was enabled to exclude unassigned and singly charged ions and the dynamic exclusion duration was set to $20 \mathrm{~s}$. The ion accumulation time was set to $300 \mathrm{~ms}$ (MS) and $50 \mathrm{~ms}$ (MS/MS). The collision energy was set to $35 \%$, and one microscan was acquired for each spectrum. For all LC-MS measurements, singly charged ions and ions with unassigned charge state were excluded from triggering MS2 events.

To determine changes in protein expressions across samples, a MS1-based label-free quantification was carried out. Therefore, the generated raw files were imported into the Progenesis QI software (Nonlinear Dynamics, Version 2.0) and analyzed using the default parameter settings. MS/MS-data were exported directly from Progenesis QI in mgf format and searched against a decoy database of the forward and reverse sequences of the predicted proteome from Caulobacter crescentus (strain NA1000/CB15N) (Uniprot, download date: 08/09/2015, total of (8234 entries) using MASCOT. The search criteria were set as following: full tryptic specificity was required (cleavage after lysine or arginine residues); three missed cleavages were allowed; carbamidomethylation (C) was set as fixed modification; oxidation (M) as variable modification. The mass tolerance was set to $10 \mathrm{ppm}$ for precursor ions and to $0.6 \mathrm{Da}$ for fragment ions. Results from the database search were imported into Progenesis QI and the final peptide measurement list containing the peak areas of all identified peptides, respectively, was exported. This list was further processed and statically analyzed using our in-house developed SafeQuant R script (SafeQuant, https://github.com/eahrne/SafeQuant ${ }^{34}$ ). The peptide and protein false discovery rate was set to $1 \%$ using the number of reverse hits in the dataset. All quantitative analyses were performed in biological triplicates. All raw data and results associated with the manuscript have been deposited to the ProteomeXchange Consortium via the PRIDE ${ }^{35}$ partner repository with the dataset identifier PXD012739.

Genetic selection for c-di-GMP-independent mutations in shkA. Six independent colonies of strain AKS1 (CB15 rcdG ${ }^{0}$ AlacA:: $\Omega$ ampG::pNPTStet-ampG) harboring in addition plasmid pAK503-spmX were inoculated into $5 \mathrm{ml}$ PYE containing chloramphenicol $\left(1 \mathrm{mgl}^{-1}\right)$ and oxytetracycline $\left(10 \mathrm{mgl}^{-1}\right)$ and grown overnight. For UV mutagenesis, $2 \mathrm{ml}$ of each culture was distributed in six-well plates and irradiated with 10,000 mJ UV light using a Stratalinker, after which $5 \mathrm{ml}$ PYE containing chloramphenicol $\left(1 \mathrm{mgl}^{-1}\right)$ and oxytetracycline $\left(10 \mathrm{mg} \mathrm{l}^{-1}\right)$ were added and cultures grown for $7 \mathrm{~h}$ at $30^{\circ} \mathrm{C}$ with shaking. Five hundred microliters of each culture were plated on PYE plates containing chloramphenicol $\left(1 \mathrm{mgl}^{-1}\right)$, oxytetracycline $\left(10 \mathrm{mgl}^{-1}\right)$, and kanamycin $\left(6.25 \mathrm{mg} \mathrm{l}^{-1}\right)$ and plates incubated at $30^{\circ} \mathrm{C}$ for 4 days. All colonies from one plate were pooled, diluted to an $\mathrm{OD}_{660}$ of 0.1 and grown for $7 \mathrm{~h}$ at $30^{\circ} \mathrm{C}$, and phage lysates were prepared for each independent pool. Following transduction of strain AKS17 (CB15 $\mathrm{rcdG}^{0} \Delta l a c A:: \Omega$ /pAK502-spmX) with individual pool lysates, cells were plated on PYE containing chloramphenicol $(1 \mathrm{mg} / \mathrm{l})$, oxytetracycline $\left(10 \mathrm{mgl}^{-1}\right)$, and X-Gal $\left(40 \mathrm{mgl}^{-1}\right)$ and incubated at $30^{\circ} \mathrm{C}$ for 3 days. Blue colonies were once restreaked to confirm their blue colony phenotype, followed by colony-PCR using primers 9765 and 9766 and sequencing (Microsynth, Balgach, Switzerland) of the PCR product using primers 9765, 9766, 6011, and 6498 .

\section{Genetic screen for mutations abolishing spmX'-'IacZ activity. Transposon} mutagenesis was performed by delivery of pSAM-Ec in strain UJ6168 carrying pAK502-spmX by conjugation and cells were plated on PYE containing chloramphenicol $\left(1 \mathrm{mgl}^{-1}\right)$, kanamycin $\left(50 \mathrm{mgl}^{-1}\right)$, nalidixic acid $\left(20 \mathrm{mgl}^{-1}\right)$, and $\mathrm{X}$ $\mathrm{Gal}\left(40 \mathrm{mg} \mathrm{l}^{-1}\right)$ and incubated at $30^{\circ} \mathrm{C}$ for 3 days. White colonies were pooled in $5 \mathrm{ml}$ PYE supplemented with appropriate antibiotics and grown overnight to prepare $\varphi$ CR30 lysates. From this pool lysate transposon insertions were transduced in a clean background (UJ6168/pAK502-spmX), followed by blue/white screening as described above. Lysates were prepared from single white colonies and mutations transduced in a NA1000 wild-type background. Transposon insertion sites were mapped using a two-step semi-arbitrary PCR approach ${ }^{36}$. Primers 9058 , 9003 and 9004 were used in the first PCR and primers 9058 and 9005 in the second PCR. PCR products were sequenced (Microsynth, Balgach, Switzerland) with 
primer 9058. In one strain (AKS50), the transposon mapped within pleD at position $2^{\prime} 695^{\prime} 825$ of the NA1000 reference genome ${ }^{37}$.

Protein expression and purification. For isothermal titration calorimetry (ITC) and in vitro phosphorylation experiments shown in Fig. 2, proteins were expressed and purified as follows. E. coli Rosetta 2(DE3) cells were used to express proteins from pET28a and pET32b expression plasmids. Cells were grown in LB-Miller supplemented with the appropriate antibiotics to an $\mathrm{OD}_{600}$ of $0.4-0.6$, expression was then induced with $0.5 \mathrm{mM}$ IPTG for $4 \mathrm{~h}$ at $30^{\circ} \mathrm{C}$. Proteins were purified on an ÄKTApurifier 10 system (GE Healthcare) using $1 \mathrm{ml}$ HisTrap HP columns (GE Healthcare) followed by size exclusion chromatography (HiLoad 16/60 Superdex 200) using the following buffers: lysis buffer (wash buffer supplemented with protease inhibitor, DNase I [NEB]), wash buffer (20 mM HEPES-KOH pH 8.0, $0.5 \mathrm{M} \mathrm{NaCl}, 10 \%$ glycerol, $20 \mathrm{mM}$ imidazole, $1 \mathrm{mM}$ DTT), elution buffer (20 mM HEPES-KOH pH 8.0 $0.5 \mathrm{M} \mathrm{NaCl}, 10 \%$ glycerol, $500 \mathrm{mM}$ imidazole, $1 \mathrm{mM}$ DTT), storage buffer (10 mM HEPES-KOH pH 8.0, $50 \mathrm{mM} \mathrm{KCl,} \mathrm{10 \%} \mathrm{glycerol,} 0.1 \mathrm{mM}$ EDTA, $1 \mathrm{mM}$ DTT). $\mathrm{MgCl}_{2}$ was added to reaction mixtures immediately prior to experiments to a final concentration of $5 \mathrm{mM}$. For all other experiments using purified ShkA, mutant variants thereof and $\mathrm{ShkA} \mathrm{A}_{\mathrm{REC} 1}$, except NMR experiments (see below), proteins were expressed in E. coli BL21(DE3) grown in LB-Miller at $37^{\circ} \mathrm{C}$ in $500-\mathrm{ml}$ cultures, with IPTG induction $(1 \mathrm{mM})$ at an $\mathrm{OD}_{600}$ of $0.5-0.8$ followed by incubation for $4 \mathrm{~h}$. Cells were harvested by centrifugation $\left(5000 \times g, 20 \mathrm{~min}, 4^{\circ} \mathrm{C}\right)$, washed once with $20 \mathrm{ml}$ of $1 \mathrm{X}$ $\mathrm{PBS}$, flash-frozen in liquid $\mathrm{N}_{2}$, and stored at $-80^{\circ} \mathrm{C}$ until purification. For purification, the pellet was resuspended in $8 \mathrm{ml}$ of buffer A (2X PBS containing $500 \mathrm{mM}$ $\mathrm{NaCl}, 10 \mathrm{mM}$ imidazole and $2 \mathrm{mM} \beta$-mercaptoethanol) supplemented with DNaseI (AppliChem) and Complete Protease inhibitor (Roche). After one passage through a French press cell, the suspension was ultra-centrifuged $\left(100,000 \times g, 30 \mathrm{~min}, 4^{\circ} \mathrm{C}\right)$ and the supernatant was mixed with $800 \mu \mathrm{l}$ of Ni-NTA slurry, prewashed with buffer A, and incubated for $1-2 \mathrm{~h}$ on a rotary wheel at $4{ }^{\circ} \mathrm{C}$. Ni-NTA agarose was loaded on a polypropylene column and washed with at least $50 \mathrm{ml}$ of buffer $\mathrm{A}$, after which the protein was eluted with $2.5 \mathrm{ml}$ of buffer A containing $500 \mathrm{mM}$ imidazole. The eluate was immediately loaded on a PD-10 column pre-equilibrated with kinase buffer (10 mM HEPES-KOH pH 8.0, $50 \mathrm{mM} \mathrm{KCl,} \mathrm{10 \%} \mathrm{glycerol,} 0.1 \mathrm{mM}$ EDTA, $5 \mathrm{mM}$ $\mathrm{MgCl}_{2}, 5 \mathrm{mM} \beta$-mercaptoethanol). The protein was then eluted with $3.5 \mathrm{ml}$ of kinase buffer and stored at $4{ }^{\circ} \mathrm{C}$ until further use, usually no longer than one week. Uniformly $\left[{ }^{13} \mathrm{C},{ }^{15} \mathrm{~N}\right]$-labeled protein for NMR studies was prepared by growing BL21(DE3) cells harboring pET28a-shkAREC1 in $11 \mathrm{M} 9$ minimal medium, with $1 \mathrm{~g}{ }^{15} \mathrm{NH} 4 \mathrm{Cl}$ and $2 \mathrm{~g}$ $\left[U_{-}{ }^{13} \mathrm{C}\right]$ glucose per liter medium, at $37^{\circ} \mathrm{C}$. Expression was induced at an $\mathrm{OD}_{600}$ of 0.8 with $1 \mathrm{mM}$ IPTG and cells were harvested $4 \mathrm{~h}$ post induction. ShkA $\mathrm{REC}_{\mathrm{R} 1}$ was purified using Ni-NTA slurry as described above, followed by size exclusion chromatography (HiLoad 16/60 Superdex 200) on a ÄKTApurifier 10 system (GE Healthcare) with NMR buffer ( $25 \mathrm{mM}$ Tris pH 7.2 with $50 \mathrm{mM} \mathrm{KCl,} 2 \mathrm{mM} \mathrm{MgSO}_{4}$ ) and concentration of the sample using Vivaspin6 $5 \mathrm{kDa}$ MWCO concentrators (Sartorius).

Isothermal titration calorimetry (ITC). ITC binding assays were performed with a VP-ITC isothermal titration calorimeter (MicroCal). Concentrations were $12 \mu \mathrm{M}$ ShkA in the cell and $130 \mu \mathrm{M}$ cdG in the syringe. ITC Buffer: $10 \mathrm{mM}$ HEPES-KOH $\mathrm{pH} 8.0,100 \mathrm{mM} \mathrm{NaCl}, 10 \%$ glycerol, $1 \mathrm{mM}$ DTT. After a first injection of $3 \mu \mathrm{l}, 10 \mu \mathrm{l}$ was injected at 29 time points. Data analysis was performed with MicroCal (ORIGIN) and fitted with the One binding site model of ORIGIN.

In vitro phosphorylation assays. Kinase assays were adapted from ${ }^{9}$. Reactions were performed in kinase buffer supplemented with $433 \mu \mathrm{M}$ ATP and 5-20 $\mu \mathrm{Ci}$ $\left[\gamma^{32} \mathrm{P}\right]$-ATP $(3000 \mathrm{Ci} / \mathrm{mmol}$, Hartmann Analytic) at room temperature if not otherwise mentioned. Additional nucleotides were added as indicated in the figures or figure legends. Kinase reactions contained $5 \mu \mathrm{M}$ ShkA and were run for $3.5 \mathrm{~min}$ at room temperature if not otherwise mentioned and stopped by addition of $5 \mathrm{X}$ SDS sample buffer and stored on ice, then run on 12.5\% SDS-PAGE or precast Mini-Protean TGX (Biorad) gels. Wet gels were exposed to a phosphor screen for $0.5-3 \mathrm{~h}$ and then scanned using a Typhoon FLA 7000 imaging system (GE Healthcare), after which gels were stained with Coomassie Brilliant Blue.

C-di-GMP UV-crosslinking assays. ${ }^{32} \mathrm{P}$-labeled c-di-GMP was prepared by incubation of $250 \mu \mathrm{Ci}$ [ $\mathrm{a}^{32} \mathrm{P}$ ]-GTP $\left(3000 \mathrm{Ci} \mathrm{mmol}^{-1}\right.$, Hartmann Analytic) in $200 \mu \mathrm{l}$ DgcZ reaction buffer ( $50 \mathrm{mM}$ Tris- $\mathrm{HCl} \mathrm{pH} 7.5,50 \mathrm{mM} \mathrm{NaCl}, 5 \mathrm{mM} \mathrm{MgCl}$ ) with $2 \mu \mathrm{M}$ purified $\mathrm{DgcZ}$ for $24 \mathrm{~h}$ at $30^{\circ} \mathrm{C}$. DgcZ (YdeH) was purified as described previously ${ }^{38}$. The reaction was stopped by boiling and, after centrifugation ( $1 \mathrm{~min}$, $16,000 \times g)$, the supernatant containing radiolabeled c-di-GMP was removed and stored at $-20^{\circ} \mathrm{C}$. Non-radio-labeled c-di-GMP was prepared as described previously ${ }^{38}$ with the following modifications. $5 \%(\mathrm{v} / \mathrm{v})$ methanol and $5 \mathrm{mM}$ tetraethylammonium bromide were added to the c-di-GMP reaction and the mixture was loaded on a Luna $10 \mu \mathrm{M}$ C18(2) $100 \AA$, $100 \times 21.2 \mathrm{~mm}$ column (Phenomenex). GTP was removed by isocratic elution in TEAB buffer $(5 \mathrm{mM}$ tetraethylammonium bromide, $5 \%[\mathrm{v} / \mathrm{v}]$ methanol) over three column volumes $(\mathrm{CV})$, after which c-diGMP was eluted with a linear gradient from 0-20\% ethanol in TEAB buffer over four CV. c-di-GMP was lyophilized overnight, resuspended in $\mathrm{ddH}_{2} \mathrm{O}$ at a concentration of $10 \mathrm{mM}$ and aliquoted. Aliquots were lyophilized and stored at

$-20^{\circ} \mathrm{C}$. $\mathrm{ddH}_{2} \mathrm{O}$ was added to aliquots to give a $10 \mathrm{mM}$ working stock, the concentration of which was verified by measuring absorbance at $253 \mathrm{~nm}$. A $80 \mathrm{mM}$ stock solutions of hot c-di-GMP was prepared by mixing $10-20 \mu \mathrm{Ci}{ }^{32} \mathrm{P}$-labeled cdi-GMP with $4 \mu \mathrm{l} 1 \mathrm{mM}$ cold c-di-GMP in a final volume of $50 \mu \mathrm{l}$. More dilute stock solutions were prepared by mixing with $\mathrm{ddH}_{2} \mathrm{O}$. UV-crosslinking reactions $(16 \mu \mathrm{l})$ contained $4 \mu \mathrm{l}$ of hot c-di-GMP to give the indicated final concentrations and $0.5 \mu \mathrm{M}$ ShkA, ShkA mutant variants or ShkA $A_{\mathrm{REC} 1}$ in kinase buffer, and were incubated at room temperature for $45 \mathrm{~min}$ before UV crosslinking at $4{ }^{\circ} \mathrm{C}, 254 \mathrm{~nm}$, $2 \mathrm{~min}$ on a Caprotec Protein Detector. For competition experiments, reactions contained a 50-fold molar excess of cold c-di-GMP over hot c-di-GMP added from a $10 \mathrm{mM}$ stock. Samples were mixed with 5X SDS sample buffer, boiled for $10 \mathrm{~min}$ and run on $12.5 \%$ SDS-PAGE or precast Mini-Protean TGX (Biorad) gels. Gels were stained with Coomassie Brilliant Blue, dried and exposed to a phosphor screen that was scanned using a Typhoon FLA 7000 imaging system (GE Healthcare). Bands of autoradiographs were quantified using Fiji and normalized to wild-type ShkA, which was run in parallel to mutant variants or ShkA $A_{\mathrm{RECl}}$ on the same gel for each experiment. Experiments were repeated twice and values represent means and standard deviations. Binding curves were fitted to a "Binding-saturation, One site-Total" model using Prism 7 (GraphPad)

Nuclear magnetic resonance (NMR) spectroscopy. All NMR spectra were recorded at $25^{\circ} \mathrm{C}$ on a Bruker Avance-700 spectrometer equipped with a cryogenically cooled triple-resonance probe. ShkA $\mathrm{REC}_{1}$ protein samples were prepared in $25 \mathrm{mM}$ Tris pH 7.2 with $50 \mathrm{mM} \mathrm{KCl}$ and $2 \mathrm{mM} \mathrm{MgSO}_{4}$ in $95 \% / 5 \% \mathrm{H}_{2} \mathrm{O} / \mathrm{D}_{2} \mathrm{O}$. For the sequence-specific backbone resonance assignment of $950 \mu \mathrm{M}\left[\mathrm{U}_{-}{ }^{15} \mathrm{~N},{ }^{13} \mathrm{C}\right]-$ ShkA $A_{R E C l}$, the following NMR experiments were recorded: $2 \mathrm{D}\left[{ }^{15} \mathrm{~N},{ }^{1} \mathrm{H}\right]-\mathrm{TROSY}$, 3D HNCA and 3D HNCACB. For the c-di-GMP binding experiments a series of $2 \mathrm{D}\left[{ }^{15} \mathrm{~N},{ }^{1} \mathrm{H}\right]-\mathrm{TROSY}$ spectra of $200 \mu \mathrm{M}\left[U_{-}{ }^{15} \mathrm{~N}\right]-S h k A_{\mathrm{REC} 1}$ were recorded with c-di-GMP concentrations of $0 \mu \mathrm{M}, 20 \mu \mathrm{M}, 50 \mu \mathrm{M}, 100 \mu \mathrm{M}, 150 \mu \mathrm{M}$, $200 \mu \mathrm{M}$ and $400 \mu \mathrm{M}$. CSPs $(\Delta \delta(\mathrm{HN}))$ of amide moieties were calculated as $\Delta \delta(\mathrm{HN})=\sqrt{0.5 \cdot\left(\Delta \delta\left({ }^{1} \mathrm{H}\right)^{2}+\left(0.2 \cdot \Delta \delta\left({ }^{15} \mathrm{~N}\right)\right)^{2}\right)}$, where $\Delta \delta\left({ }^{1} \mathrm{H}\right)$ and $\Delta \delta\left({ }^{15} \mathrm{~N}\right)$, are the amide proton and amide nitrogen chemical shift differences to the reference spectrum, respectively. Combined secondary chemical shifts of $\mathrm{C} \alpha$ and $\mathrm{C} \beta$ were calculated relative to the random-coil values of Kjaergaard and Poulson ${ }^{39}$.

C-di-GMP extraction and quantifications. Strains AKS371 and AKS412 were grown in $2 \mathrm{ml}$ PYE overnight, diluted 40 -fold the next day in $20 \mathrm{ml}$ PYE and grown for $6 \mathrm{~h}$ until reaching an $\mathrm{OD}_{660}$ of $\sim 0.35 .14 \mathrm{ml}$ were spun down $(11,000 \times g, 5 \mathrm{~min}$,

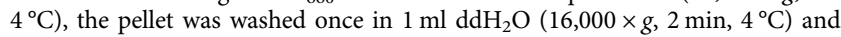
snap-frozen in liquid nitrogen. Metabolites were extracted by resuspending the pellet in $300 \mu \mathrm{l}$ ice-cold extraction solvent (40\% [v/v] acetonitrile, $40 \%[\mathrm{v} / \mathrm{v}]$ methanol, $20 \%$ [v/v] $\mathrm{ddH}_{2} \mathrm{O}$ ) and incubation at $95^{\circ} \mathrm{C}$ for $10 \mathrm{~min}$. Extractions were repeated on the pellet twice with $200 \mu \mathrm{l}$ extraction solvent and the pooled extracts $(700 \mu \mathrm{l})$ were stored overnight at $-80^{\circ} \mathrm{C}$. Remaining debris was removed by centrifugation $\left(16,000 \times g, 10 \mathrm{~min}, 4^{\circ} \mathrm{C}\right)$, the supernatant was transferred to a fresh microcentrifuge tube and solvent was removed using a Speedvac. LC-MS-based cdi-GMP quantification was performed by the Zentrale Forschungseinrichtung (ZFA) Metabolomics, Medizinische Hochschule Hannover, Germany, and cellular concentrations were calculated as described before ${ }^{11}$. Experiments were performed in biological triplicates and values given are means and standard deviations.

Sequence alignments, structure prediction, and modeling. The $C$. crescentus ShkA sequence was blasted against the nonredundant protein sequences (nr) database using default settings and the first 500 hits were aligned in Geneious using the Geneious Alignment algorithm with default settings (Supplementary Data 1). The alignment was manually checked for the presence of the DDR motif in the REC1-REC2 linker and the sequences that harbored this motif (see Fig. S3A) were realigned as described above. This alignment was used to create sequence logos using WebLogo $3^{40}$, construct a phylogenetic tree using Geneious Tree Builder with default settings and calculate conservation scores using the ConSurf server ${ }^{41}$. Four proteins of the original blast set that do not contain the DDR motif were included in the phylogenetic tree for comparison. Secondary structure prediction for E. coli BarA $_{\mathrm{PRD}}$ (residues 532-655) was performed with HHPred ${ }^{42}$ and the ShkA $\mathrm{REC1}^{-}$ BarA $A_{\mathrm{PDR}}$ alignment was generated in Geneious as described above. The ShkA $\mathrm{REC1}^{-}$ $3 \mathrm{GRC}$ alignment was generated using HHPred. The ShkA $\mathrm{REC1}_{\mathrm{B}}$ structure was modeled using the SWISS-MODEL workspace ${ }^{43}$ with PDB entry 3 GRC as the template. CSPs and conservation scores mapped onto the ShkA $A_{\mathrm{RECl}}$ model were visualized using MacPyMol (PyMOL v1.7.6.6, Schrödinger LLC).

\section{Data availability}

The sequence-specific backbone resonance assignment of the ShkARecl domain has been submitted to the Biological Magnetic Resonance Data Bank under the following accession code: 27768 [http://www.bmrb.wisc.edu/data_library/summary/index.php? bmrbId $=27768$ ]. All proteomics raw data and results associated with the manuscript have been deposited to the ProteomeXchange Consortium via the PRIDE partner repository with the dataset identifier PXD012739 [http://www.ebi.ac.uk/pride/archive/ projects/PXD012739]. The source data underlying Figs. 1d-h, 2c-i, 3c, e-h and 5b-f and Supplementary Figs. 1b, 2a, c-e, 4 b, h and $8 \mathrm{a}$ are provided as Source Data files. The source data underlying Fig. $4 \mathrm{c}$ and Supplementary Fig. $7 \mathrm{c}$ are deposited on Zenodo [https://doi.org/10.5281/zenodo.3574247]. 
Received: 29 June 2019; Accepted: 13 January 2020;

Published online: 10 February 2020

\section{References}

1. Wang, J. D. \& Levin, P. A. Metabolism, cell growth and the bacterial cell cycle. Nat. Rev. Microbiol. 7, 822-827 (2009).

2. Cooper, S. \& Helmstetter, C. E. Chromosome replication and the division cycle of Escherichia coli B/r. J. Mol. Biol. 31, 519-540 (1968).

3. Wallden, M., Fange, D., Lundius, E. G., Baltekin, Ö. \& Elf, J. The synchronization of replication and division cycles in individual $E$. coli cells. Cell 166, 729-739 (2016).

4. Gorbatyuk, B. \& Marczynski, G. T. Regulated degradation of chromosome replication proteins DnaA and CtrA in Caulobacter crescentus. Mol. Microbiol. 55, 1233-1245 (2005).

5. Lesley, J. A. \& Shapiro, L. SpoT regulates DnaA stability and initiation of DNA replication in carbon-starved Caulobacter crescentus. J. Bacteriol. 190, 6867-6880 (2008).

6. Biondi, E. G. et al. Regulation of the bacterial cell cycle by an integrated genetic circuit. Nature 444, 899-904 (2006).

7. Tsokos, C. G., Perchuk, B. S. \& Laub, M. T. A dynamic complex of signaling proteins uses polar localization to regulate cell-fate asymmetry in Caulobacter crescentus. Dev. Cell. 20, 329-341 (2011).

8. Paul, R. et al. Allosteric regulation of histidine kinases by their cognate response regulator determines cell fate. Cell 133, 452-461 (2008).

9. Lori, C. et al. Cyclic di-GMP acts as a cell cycle oscillator to drive chromosome replication. Nature 523, 236-239 (2015).

10. Paul, R. Cell cycle-dependent dynamic localization of a bacterial response regulator with a novel di-guanylate cyclase output domain. Genes Dev. 18, 715-727 (2004).

11. Abel, S. et al. Bi-modal distribution of the second messenger c-di-GMP controls cell fate and asymmetry during the Caulobacter cell cycle. PLoS Genet. 9, e1003744 (2013).

12. Radhakrishnan, S. K., Thanbichler, M. \& Viollier, P. H. The dynamic interplay between a cell fate determinant and a lysozyme homolog drives the asymmetric division cycle of Caulobacter crescentus. Genes Dev. 22, 212-225 (2008).

13. Biondi, E. G. et al. A phosphorelay system controls stalk biogenesis during cell cycle progression in Caulobacter crescentus. Mol. Microbiol. 59, 386-401 (2006).

14. Janakiraman, B., Mignolet, J., Narayanan, S., Viollier, P. H. \& Radhakrishnan, S. K. In-phase oscillation of global regulons is orchestrated by a pole-specific organizer. Proc. Natl Acad. Sci. 113, 12550-12555 (2016).

15. Aldridge, P., Paul, R., Goymer, P., Rainey, P. \& Jenal, U. Role of the GGDEF regulator PleD in polar development of Caulobacter crescentus. Mol. Microbiol. 47, 1695-1708 (2003).

16. Joshi, K. K., Bergé, M., Radhakrishnan, S. K., Viollier, P. H. \& Chien, P. An adaptor hierarchy regulates proteolysis during a bacterial cell cycle. Cell 163, 419-431 (2015).

17. Duerig, A. et al. Second messenger-mediated spatiotemporal control of protein degradation regulates bacterial cell cycle progression. Genes Dev. 23, 93-104 (2009).

18. Gurskaya, N. G. et al. Engineering of a monomeric green-to-red photoactivatable fluorescent protein induced by blue light. Nat. Biotechnol. 24, 461-465 (2006).

19. Zähringer, F., Lacanna, E., Jenal, U., Schirmer, T. \& Boehm, A. Structure and signaling mechanism of a zinc-sensory diguanylate cyclase. Structure $\mathbf{2 1}$, 1149-1157 (2013).

20. Christen, M. et al. Asymmetrical distribution of the second messenger c-diGMP upon bacterial cell division. Science 328, 1295-1297 (2010).

21. Abel, S. et al. Regulatory cohesion of cell cycle and cell differentiation through interlinked phosphorylation and second messenger networks. Mol. Cell 43, 550-560 (2011).

22. Dubey, B. N. et al. Hybrid histidine kinase activation by cyclic di-GMPmediated domain liberation. Proc. Natl Acad. Sci. https://doi.org/10.1073/ pnas.1911427117 (2019).

23. Goodman, A. L. et al. A signaling network reciprocally regulates genes associated with acute infection and chronic persistence in Pseudomonas aeruginosa. Dev. Cell 7, 745-754 (2004).

24. Lawhon, S. D., Maurer, R., Suyemoto, M. \& Altier, C. Intestinal shortchain fatty acids alter Salmonella typhimurium invasion gene expression and virulence through BarA/SirA. Mol. Microbiol. 46, 1451-1464 (2002).

25. Rogov, V. V. et al. A new structural domain in the Escherichia coli RcsC hybrid sensor kinase connects histidine kinase and phosphoreceiver domains. J. Mol. Biol. 364, 68-79 (2006).
26. Wall, E., Majdalani, N. \& Gottesman, S. The complex Rcs regulatory cascade. Annu. Rev. Microbiol. 72, 111-139 (2018).

27. Ely, B. Genetics of Caulobacter crescentus. Methods Enzymol. 204, 372-384 (1991).

28. Viollier, P. H., Sternheim, N. \& Shapiro, L. A dynamically localized histidine kinase controls the asymmetric distribution of polar pili proteins. EMBO J. 21, $4420-4428$ (2002).

29. Domian, I. J., Quon, K. C. \& Shapiro, L. Cell type-specific phosphorylation and proteolysis of a transcriptional regulator controls the G1-to-S transition in a bacterial cell cycle. Cell 90, 415-424 (1997).

30. Schindelin, J. et al. Fiji: an open-source platform for biological-image analysis. Nat. Methods 9, 676-682 (2012).

31. Ducret, A., Quardokus, E. M. \& Brun, Y. V. MicrobeJ, a tool for high throughput bacterial cell detection and quantitative analysis. Nat. Microbiol. 1, 16077 (2016).

32. Paintdakhi, A. et al. Oufti: An integrated software package for high-accuracy, high-throughput quantitative microscopy analysis. Mol. Microbiol. https://doi. org/10.1111/mmi.13264 (2015).

33. Lori, C., Kaczmarczyk, A., de Jong, I. \& Jenal, U. A single-domain response regulator functions as an integrating hub to coordinate general stress response and development in Alphaproteobacteria. mBio 9, e00809-18 (2018).

34. Ahrné, E. et al. Evaluation and improvement of quantification accuracy in isobaric mass tag-based protein quantification experiments. J. Proteome Res. 15, 2537-2547 (2016)

35. Vizcaíno, J. A. et al. 2016 update of the PRIDE database and its related tools. Nucleic Acids Res. 44, D447-D456 (2016).

36. Francez-Charlot, A., Kaczmarczyk, A. \& Vorholt, J. A. The branched CcsA/ CckA-ChpT-CtrA phosphorelay of Sphingomonas melonis controls motility and biofilm formation. Mol. Microbiol. 97, 47-63 (2015).

37. Marks, M. E. et al. The genetic basis of laboratory adaptation in Caulobacter crescentus. J. Bacteriol. 192, 3678-3688 (2010).

38. Zähringer, F., Massa, C. \& Schirmer, T. Efficient enzymatic production of the bacterial second messenger c-di-GMP by the diguanylate cyclase $\mathrm{YdeH}$ from E. coli. Appl. Biochem. Biotechnol. 163, 71-79 (2010).

39. Kjaergaard, M. \& Poulsen, F. M. Sequence correction of random coil chemical shifts: correlation between neighbor correction factors and changes in the Ramachandran distribution. J. Biomol. NMR 50, 157-165 (2011).

40. Crooks, G. E., Hon, G., Chandonia, J.-M. \& Brenner, S. E. WebLogo: a sequence logo generator. Genome Res. 14, 1188-1190 (2004).

41. Ashkenazy, H. et al. ConSurf 2016: an improved methodology to estimate and visualize evolutionary conservation in macromolecules. Nucleic Acids Res. 44, W344-W350 (2016).

42. Söding, J., Biegert, A. \& Lupas, A. N. The HHpred interactive server for protein homology detection and structure prediction. Nucleic Acids Res. 33, W244-W248 (2005).

43. Waterhouse, A. et al. SWISS-MODEL: homology modelling of protein structures and complexes. Nucleic Acids Res. 46, W296-W303 (2018).

\section{Acknowledgements}

We thank Tim Sharpe (Biophysics Facility, Biozentrum, University of Basel), Alexander Schmidt (Proteomics Core Facility, Biozentrum, University of Basel), Janine Bögli, and Stella Stefanova (FACS Core Facility, Biozentrum, University of Basel) for technical guidance, Christian Lori and Benoit-Joseph Laventie for preparation of c-di-GMP, Fabienne Hamburger for cloning and strain construction, Volkhard Kaever (Zentrale Forschungseinrichtung [ZFA] Metabolomics, Medizinische Hochschule Hannover, Germany) for c-di-GMP measurements and life science editors for editing assistance. We thank Patrick Viollier, Lucy Shapiro, Justine Collier, Joseph Chen, Matthew Malvey, Régis Hallez, and Julia Vorholt for providing antibodies, plasmids, and strains. This work was supported by the European Research Council (ERC) Advanced Research Grant (3222809) and the Swiss National Science Foundation Grants 310030B_147090 and 310030B_185372 (to U.J.) and 31003A-166652 (to T.S.) A.M.H. is a Marie Heim-Vögtlin fellow of the Swiss National Science Foundation (PMPDP3_171306) and was supported by a Human Frontier Science Program (HFSP) Long-Term Postdoctoral Fellowship (LT000779/2013) and a University of Basel Stay-on-track grant.

\section{Author contributions}

Conceptualization, A.K., A.M.H., C.vA., B.D., T.S., and U.J.; Methodology, A.K., A.M.H., C.vA., J.N., R.B., S.H., and U.J.; Formal analysis, A.K., A.M.H., C.vA., R.B., S.H., and U.J.; Investigation, A.K., A.M.H., C.vA., J.N., R.B., S.H., and U.J.; Resources, B.D and T.S.; Writing-original draft, A.K., A.M.H., C.vA., R.B., S.H., and U.J. with contributions from all other authors; Funding acquisition, A.M.H., S.H., T.S., and U.J.

\section{Competing interests}

The authors declare no competing interests. 


\section{Additional information}

Supplementary information is available for this paper at https://doi.org/10.1038/s41467020-14585-6.

Correspondence and requests for materials should be addressed to U.J.

Peer review information Nature Communications thanks the anonymous reviewers for their contribution to the peer review of this work.

Reprints and permission information is available at http://www.nature.com/reprints

Publisher's note Springer Nature remains neutral with regard to jurisdictional claims in published maps and institutional affiliations. (c) Open Access This article is licensed under a Creative Commons Attribution 4.0 International License, which permits use, sharing, adaptation, distribution and reproduction in any medium or format, as long as you give appropriate credit to the original author(s) and the source, provide a link to the Creative Commons license, and indicate if changes were made. The images or other third party material in this article are included in the article's Creative Commons license, unless indicated otherwise in a credit line to the material. If material is not included in the article's Creative Commons license and your intended use is not permitted by statutory regulation or exceeds the permitted use, you will need to obtain permission directly from the copyright holder. To view a copy of this license, visit http://creativecommons.org/licenses/by/4.0/.

(C) The Author(s) 2020 